TUM/T39-01-16

\title{
Phases of QCD, Thermal Quasiparticles and Dilepton Radiation from a Fireball
}

\author{
Thorsten Renk ${ }^{a}$, Roland Schneider ${ }^{a}$, Wolfram Weise ${ }^{a, b}$ \\ ${ }^{a}$ Physik Department, Technische Universität München, D-85747 Garching, GERMANY \\ ${ }^{b}$ ECT $^{*}$, Villa Tambosi, I-38050 Villazzano (Trento), ITALY
}

\begin{abstract}
We calculate dilepton production rates from a fireball adapted to the kinematical conditions realized in ultrarelativistic heavy ion collisions over a broad range of beam energies. The freeze-out state of the fireball is fixed by hadronic observables. We use this information combined with the initial geometry of the collision region to follow the space-time evolution of the fireball. Assuming entropy conservation, its bulk thermodynamic properties can then be uniquely obtained once the equation of state (EoS) is specified. The high-temperature (QGP) phase is modelled by a non-perturbative quasiparticle model that incorporates a phenomenological confinement description, adapted to lattice $\mathrm{QCD}$ results. For the hadronic phase, we interpolate the EoS into the region where a resonance gas approach seems applicable, keeping track of a possible overpopulation of the pion phase space. In this way, the fireball evolution is specified without reference to dilepton data, thus eliminating it as an adjustable parameter in the rate calculations. Dilepton emission in the QGP phase is then calculated within the quasiparticle model. In the hadronic phase, both temperature and finite baryon density effects on the photon spectral function are incorporated. Existing dilepton data from CERES at 158 and $40 \mathrm{AGeV} \mathrm{Pb-Au}$ collisions are well described, and a prediction for the PHENIX setup at RHIC for $\sqrt{s}=200 \mathrm{AGeV}$ is given.
\end{abstract}

\section{Introduction}

There are convincing arguments from lattice simulations 11 that QCD undergoes a phase transition at a temperature of about $150-170 \mathrm{MeV}$ [2 from a confined hadronic phase to a phase where quarks and gluons constitute the relevant degrees of freedom, the quark-gluon plasma (QGP). It is believed that chiral symmetry restoration, associated with the vanishing of the chiral condensate $\langle\bar{q} q\rangle$, takes place at about the same temperature. This new state of matter is clearly of enormous interest, and the search for experimental evidence of its creation in ultrarelativistic heavy ion collisions (URHIC) is going on. However, it is still a matter of debate whether the QGP has already been encountered. Any signature of the QGP is folded with the time evolution (and consequently the evolution of volume, temperature and baryon density) of the fireball created in such a collision. Furthermore, this evolution continues after the phase transition and any information is thus mixed with signals from the hadronic phase. Therefore, extracting an unambiguous signal for the QGP phase is not an easy task.

Dileptons $\left(e^{+} e^{-}\right.$and $\mu^{+} \mu^{-}$pairs) are interesting probes in this context since they do not interact strongly and escape unthermalized from the hot region at all stages of the collision. Therefore, in contrast to hadronic probes which can tell only of the late stages (the freeze-out), dileptons carry information also on the early moments of the collision where the QGP phase is expected to exist. In the QGP phase,

*) work supported in part by BMBF and GSI 
dileptons originate mainly from $q \bar{q}$ annihilation processes, whereas in the hadronic phase the main sources are pion and kaon annihilation processes which are enhanced due to the formation of the light vector mesons $\rho, \omega$ and $\phi$. In order to interpret signals from the QGP, one must obviously first understand the hadronic phase.

Unfortunately, the initial conditions of a fireball formed in URHIC are not well under control, nor is its subsequent evolution known in sufficient detail so far. Different scenarios (pure hadronic, pure QGP and mixed phases) can all be designed such that they are in rough agreement with experimental data.

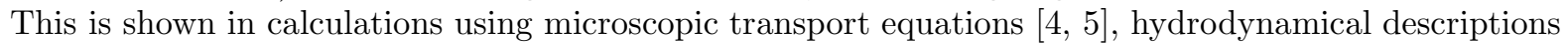
[6, 7] and simplified approaches [8, 9]. In this paper, we make attempts to tighten the constraints on the QGP equation of state (EoS) and the fireball evolution dynamics by establishing contacts with lattice QCD thermodynamics and hadronic observables. We extend the work of [10] (and other previous work) in several directions.

First, we develop a thermodynamically consistent quasiparticle picture, incorporating important aspects of confinement, to describe both the fireball dynamics and the dilepton radiation from the quark-gluon phase at the early stages of the collision. Perturbation theory is known not to work in the quark-gluon phase 11] even at temperatures well above $T_{C}$. We adopt instead an approach which treats quarks and gluons as massive thermal quasiparticles, with their properties determined to reproduce lattice QCD results. The driving force of the phase transition, the confinement/deconfinement process, is given a statistical meaning in terms of a suppression of thermally active partonic degrees of freedom as the critical temperature is approached from above. For the hadronic phase, we calculate the dependence of the photon spectral function (that enters the dilepton rate) on temperature and baryon density using Vector Meson Dominance combined with Chiral Dynamics.

Second, a fireball model reminiscent of hydrodynamics is set up. The endpoint of the fireball evolution is fixed by requiring agreement with hadronic observables, such as Hanbury-Brown-Twiss (HBT) radii, $m_{t}$-spectra and $d N / d y$ distributions, which carry information on the freeze-out stage. Information on the initial state can be gained by estimates of the nuclear overlap geometry. We then construct the evolution between initial and freeze-out conditions by following the fireball thermodynamics along volume slices of constant proper time $\tau$. This is done under the assumption of entropy conservation where the total entropy of the system is determined at freeze-out. The EoS in the QGP phase is calculated using a quasiparticle picture. In the hadronic phase a smooth interpolation to a hadronic resonance gas approach is applied. We thus obtain the temperature $T$ of the system at a given proper time. This procedure results in a thermodynamically self-consistent description of the fireball evolution, which is fixed by hadronic observables only.

Using this model as an input, we calculate dilepton rates and compare results to existing CERES/NA45 data of 40 and $160 \mathrm{AGeV} \mathrm{Pb-Au} \mathrm{collisions,} \mathrm{finding} \mathrm{good} \mathrm{agreement} \mathrm{in} \mathrm{both} \mathrm{cases.} \mathrm{We} \mathrm{also} \mathrm{give} \mathrm{predictions}$ for the kinematical conditions at RHIC. Differences between the present approach and other works are discussed.

\section{Dileptons from a fireball}

The lepton pair emission rate from a hot domain populated by particles in thermal equilibrium at temperature $T$ is proportional to the imaginary part of the spin-averaged, photon self-energy, with these particles as intermediate states. The thermally excited particles annihilate to yield a time-like virtual photon with four-momentum $q$ which decays subsequently into a lepton-antilepton pair. The differential pair production rate is given by

$$
\frac{d N}{d^{4} x d^{4} q}=\frac{\alpha^{2}}{\pi^{3} q^{2}} \frac{1}{e^{\beta q^{0}}-1} \operatorname{Im} \bar{\Pi}(q, T)=\frac{\alpha^{2}}{12 \pi^{4}} \frac{R(q, T)}{e^{\beta q^{0}-1}},
$$


where $\alpha=e^{2} / 4 \pi, \beta=1 / T$, and we have neglected the lepton masses. We have defined $\bar{\Pi}(q)=-\Pi_{\mu}^{\mu} / 3$ and introduce the averaged photon spectral function $R(q, T)=\left(12 \pi / q^{2}\right) \operatorname{Im} \bar{\Pi}(q, T)$. Here $\Pi^{\mu}{ }_{\mu}$ denotes the trace over the thermal photon self-energy which is equivalent to the thermal current-current correlation function

$$
\Pi_{\mu \nu}(q, T)=i \int d^{4} x e^{i q x}\left\langle\mathcal{T} j_{\mu}(x) j_{\nu}(0)\right\rangle_{\beta}
$$

where $j_{\mu}$ is the electromagnetic current. Eq.(11) is valid to order $\alpha$ in the electromagnetic interaction and to all orders in the strong interaction.

In order to compare with experimental data, we construct a model for the space-time evolution of a heavy-ion collision, assuming approximate thermal equilibrium to be a useful concept [13]. Some recent discussions suggest 14, 15] that equilibration times at the conditions of heavy ion collisions at SPS and RHIC may indeed be very short (possibly less than $1 \mathrm{fm} / \mathrm{c}$ ), small compared to expansion times of order $10-20 \mathrm{fm} / \mathrm{c}$, although this is still a matter of debate. The proper approach for our purposes is to use a simplified fireball model which employs a hot cylinder, expanding isentropically both in the longitudinal and transverse direction. Temperature and density are assumed to be spatially homogeneous at a given proper time. In chapter 5 , this fireball model is explained in detail.

The differential rate of eq.(1) is integrated over the space-time history of the collision to compare the calculated dilepton rates with the CERES/NA45 data 49] taken in Pb-Au collisions at $160 \mathrm{AGeV}$ (corresponding to a c.m. energy of $\sqrt{s} \sim 17 \mathrm{AGeV})$ and $40 \mathrm{AGeV}(\sqrt{s} \sim 8 \mathrm{AGeV})$. The CERES experiment is a fixed-target experiment. In the lab frame, the CERES detector covers the limited rapidity interval $\eta=2.1-2.65$, i.e. $\Delta \eta=0.55$. We integrate the calculated rates over the transverse momentum $p_{T}$ and average over $\eta$, given that $d^{4} p=M p_{T} d M d \eta d p_{T} d \theta$. The formula for the space-timeand $p$-integrated dilepton rates hence becomes

$$
\frac{d^{2} N}{d M d \eta}=\frac{2 \pi M}{\Delta \eta} \int_{0}^{\tau_{f}} d \tau \int d \eta V(\eta, T(\tau)) \int_{0}^{\infty} d p_{T} p_{T} \frac{d N\left(T(\tau), M, \eta, p_{T}\right)}{d^{4} x d^{4} p} \operatorname{Acc}\left(M, \eta, p_{T}\right),
$$

where $\tau_{f}$ is the freeze-out proper time of the collision, $V(\eta, T(\tau))$ describes the proper time evolution of volume elements moving at different rapidities and the function $\operatorname{Acc}\left(M, \eta, p_{T}\right)$ accounts for the experimental acceptance cuts specific to the detector. At the CERES experiment, each electron/positron track is required to have a transverse momentum $p_{T}>0.2 \mathrm{GeV}$, to fall into the rapidity interval $2.1<\eta<2.65$ in the lab frame and to have a pair opening angle $\Theta_{e e}>35 \mathrm{mrad}$. Eq.(3) is then convoluted with the finite energy resolution of the detector. Finally, for comparison with the CERES data, the resulting rate is divided by $d N_{c h} / d \eta$, the rapidity density of charged particles.

RHIC operates as a collider experiment, so in this case the fireball is centered around $\eta=0$. Here, the PHENIX detector acceptance can be schematically modelled by requiring that each electron/positron track falls in the rapidity interval $-0.35<\eta<0.35$, has transverse momentum $p_{T}>0.2 \mathrm{GeV}$ and a pair opening angle of $\Theta_{e \bar{e}}>35 \mathrm{mrad}$. At present, an abundance of data on Au-Au collisions at $\sqrt{s}=130$ $\mathrm{AGeV}$ have already been analysed, and first data of the run at the higher energy $\sqrt{s}=200 \mathrm{AGeV}$ are available.

\section{Quasiparticle description of the quark-gluon phase}

In order to proceed, we need to develop a picture of the QCD dynamics in the quark-gluon plasma phase close to the phase transition where the system presumably spends an appreciable fraction of its time in URHIC at current kinematical conditions. A realistic equation of state is needed to model the expanding 
fireball, and the calculation of the differential dilepton emission rate requires the photon self-energy with partonic intermediate states.

Since it is expected that $\alpha_{s}(T) \rightarrow 0$ at very high temperatures, one might naively expect that a perturbative approach to calculate these quantities is possible. However, it is now well-known that perturbation theory breaks down for gauge theories at finite temperature because of the occurrence of infrared divergences [11. The Hard Thermal Loop (HTL) calculation scheme consistently resums all contributions to a given order in the gauge coupling constant $g_{s}$ and is explicitly gauge invariant. Its application relies on a scale separation into 'hard' $(p \sim T)$ and 'soft' $\left(p \sim g_{s} T\right)$ momentum modes with $g_{s} \ll 1$, though for QCD, this condition is likely to be fulfilled only at extremely large $T$ far outside the scope of present and future experiments [12]. In addition, non-abelian gauge theories also suffer from a dependence on an 'ultra-soft' magnetic mass $m_{m a g} \sim g_{s}^{2} T$ which is intrinsically nonperturbative.

The imaginary part of the photon self-energy $\operatorname{Im} \bar{\Pi}$ appearing in eq.(1) has been calculated to two-loop order in thermal perturbation theory [17], and there exist estimates for the three-loop contributions [18]. So far, the convergence of the resulting series is badly behaved even for small couplings $g_{s}$. A similar behaviour has been found in the perturbative calculation of the free energy of a QGP and also in simple scalar theory. Furthermore, close to $T_{C}$ we expect intrinsically non-perturbative dynamics to enter: the confinement/deconfinement transition and chiral symmetry breaking are not amenable in an expansion in $g_{s}$. In the light of these facts, we will use a more phenomenological approach to QCD thermodynamics in this work.

One way to obtain nonperturbative input is through finite temperature lattice simulations of a pure gluonic or quark-gluon plasma. We have shown recently that it is possible to describe the equation of state (EoS) of such systems to a very good approximation by the EoS of a gas of quasiparticles with thermally generated masses, incorporating confinement phenomenologically by a temperature-dependent effective number of active degrees of freedom. Here we give a short summary of the method and refer the reader to [19] for a more detailed discussion.

From asymptotic freedom, we expect that at extremely high temperatures the plasma consists of quasifree quarks and gluons. HTL perturbative calculations find, for thermal momenta, spectral functions of the form $\delta\left(E^{2}-k^{2}-m^{2}(T)\right)$ with $m(T) \sim g_{s} T$. As long as the spectral functions of the thermal excitations at lower temperatures resemble qualitatively this asymptotic form, a quasiparticle description is expected to be applicable. The QCD dynamics is then incorporated in the thermal masses of the 'effective' quarks and gluons and in a function $B(T)$ which is necessary for thermodynamical consistency and plays the rôle of the thermal vacuum energy.

Since the existence of a preferred frame of reference (in which the hot matter is at rest) breaks Lorentz invariance, new partonic excitations (longitudinal gluonic plasmons and helicity-flipped plasminos) are also present in the plasma. However, their spectral strengths are exponentially suppressed for high temperatures and momenta $k \sim T$ which dominate the integrals of macroscopic thermodynamic quantities such as pressure, entropy and energy density, therefore their contributions can be neglected. This is also in line with results from a lattice calculation of the quark and gluon propagators [16]. For the differential dilepton rate, their influence is not so easily discarded, and we will discuss that in section 4 .

The thermal excitations can then be described by a dispersion equation

$$
E^{2}(k, T)=k^{2}+m_{i}^{2}(T) .
$$

Here, $k=|\mathbf{k}|$, and the subscript $i$ labels the particle species: $i=g$ for gluons and $i=q$ for quarks. $m_{i}(T)$ stands for a thermal mass which is derived from the self-energy of the corresponding particle, evaluated at thermal momenta $E, k \sim T$. This is expected to be meaningful as long as the self-energy is only weakly momentum dependent in that kinematic region. Additionally, for a quasiparticle to be a meaningful concept at all, we require the imaginary part of the self-energy, and hence its thermal width, to be small. 
The gluon mass, following ref. 19, becomes

$$
\frac{m_{g}(T)}{T}=\sqrt{\frac{N_{C}}{6}+\frac{N_{f}}{12}} \tilde{g}\left(T, N_{C}, N_{f}\right)
$$

with the effective coupling specified as

$$
\tilde{g}\left(T, N_{C}, N_{f}\right)=\frac{g_{0}}{\sqrt{11 N_{C}-2 N_{f}}}\left([1+\delta]-\frac{T_{C}}{T}\right)^{\gamma} .
$$

$N_{C}$ and $N_{f}$ stand for the number of colours and flavours, respectively. The functional dependence of $m_{g}(T)$ on $T$ is based on the conjecture that the phase transition is weakly first order or second order which suggests an almost powerlike behaviour $m \sim\left(T-T_{C}\right)^{\gamma}$ with some pseudo-critical exponent $\gamma>0$. Setting $g_{0}=9.4, \delta=10^{-6}$ and $\gamma=0.1$, the effective mass (5) approaches the HTL result at high temperatures.

The thermal quark mass reads

$$
\frac{m_{q}(T)}{T}=\sqrt{\left(\frac{m_{q, 0}}{T}+\frac{1}{4} \sqrt{\frac{N_{C}^{2}-1}{N_{C}}} \tilde{g}(T)\right)^{2}+\frac{N_{C}^{2}-1}{16 N_{C}} \tilde{g}(T)^{2}}
$$

with the zero-temperature bare quark mass $m_{q, 0}$. Note that in contrast to previous quasiparticle models extrapolated from HTL calculations [20, 21, the thermal masses used here drop as $T_{C}$ is approached from above. Of course, for $T \gg T_{C}$, the near-critical behaviour inferred in (6) ceases to be valid and the perturbative limit of $m_{g}(T)$ and $m_{q}(T)$ will be recovered.

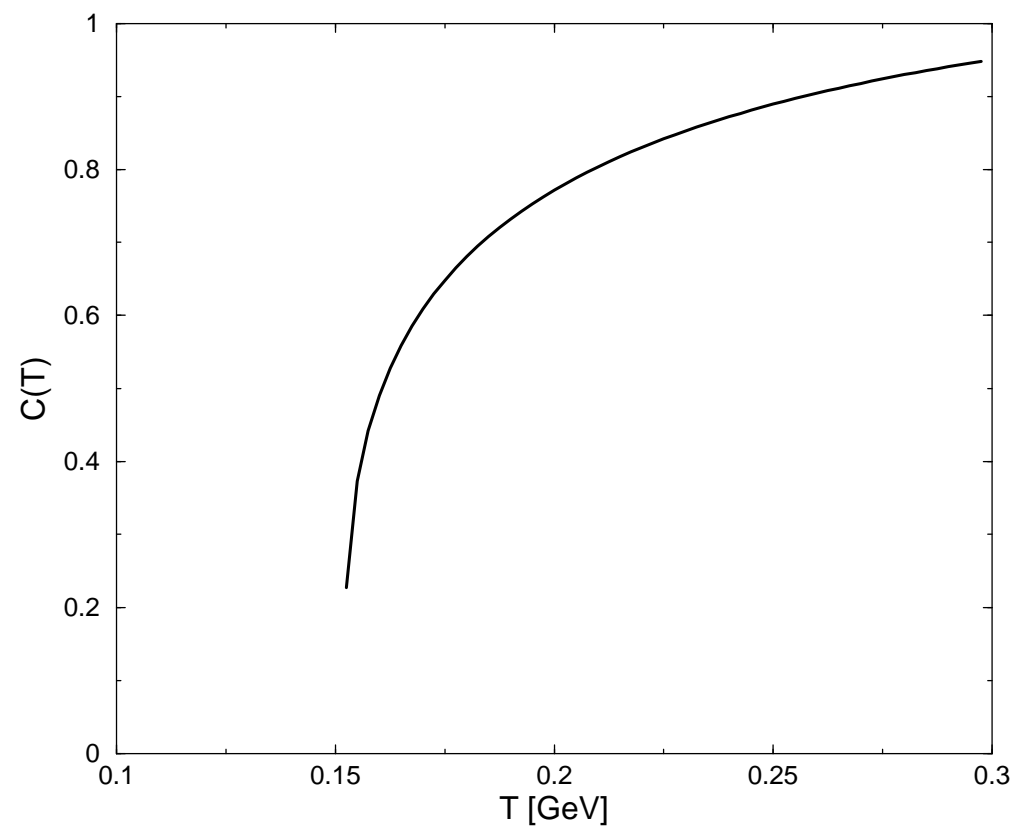

Figure 1: The confinement factor $C(T)$ as a function of temperature for two light flavours $\left(m_{u, d}=0\right)$ and one heavier flavour $\left(m_{s} \simeq 170 \mathrm{MeV}\right)$. 
The pressure of the QGP system takes the following form:

$$
p(T)=\frac{\nu_{g}}{6 \pi^{2}} \int_{0}^{\infty} d k\left[C(T) f_{B}\left(E_{k}^{g}\right)\right] \frac{k^{4}}{E_{k}^{g}}+\sum_{i=1}^{N_{f}} \frac{2 N_{C}}{3 \pi^{2}} \int_{0}^{\infty} d k\left[C(T) f_{D}\left(E_{k}^{i}\right)\right] \frac{k^{4}}{E_{k}^{i}}-B(T) .
$$

Energy density $\epsilon(T)$ and entropy density $s(T)$ follow accordingly (see 19] for details):

$$
\begin{gathered}
\epsilon(T)=\frac{\nu_{g}}{2 \pi^{2}} \int_{0}^{\infty} d k k^{2}\left[C(T) f_{B}\left(E_{k}^{g}\right)\right] E_{k}^{g}+\sum_{i=1}^{N_{f}} \frac{2 N_{C}}{\pi^{2}} \int_{0}^{\infty} d k k^{2}\left[C(T) f_{D}\left(E_{k}^{i}\right)\right] E_{k}^{i}+B(T), \\
s(T)=\frac{\nu_{g}}{2 \pi^{2} T} \int_{0}^{\infty} d k k^{2}\left[C(T) f_{B}\left(E_{k}^{g}\right)\right] \frac{\frac{4}{3} k^{2}+m_{g}^{2}(T)}{E_{k}^{g}}+\sum_{i=1}^{N_{f}} \frac{2 N_{C}}{\pi^{2} T} \int_{0}^{\infty} d k k^{2}\left[C(T) f_{D}\left(E_{k}^{i}\right)\right] \frac{\frac{4}{3} k^{2}+m_{i}^{2}(T)}{E_{k}^{i}} .
\end{gathered}
$$

Here, $E_{k}^{g}=\sqrt{k^{2}+m_{g}^{2}(T)}$ and $E_{k}^{i}=\sqrt{k^{2}+m_{i}^{2}(T)}$ for each quark flavour $q=i$. The multiplicity $\nu_{g}=16$ counts the transverse gluonic degrees of freedom. The bosonic particle distribution function reads $f_{B}(E)=(\exp (E / T)-1)^{-1}$, the fermionic one is $f_{D}(E)=(\exp (E / T)+1)^{-1}$.

The function $C(T)$ accounts, in a statistical sense, for the onset of confinement by reducing the number of thermally active degrees of freedom. Its explicit form is obtained by calculating the entropy density of the QGP with the gluon mass (5) and the quark mass (7). Dividing the lattice entropy density by this result yields $C(T)$. It can be parametrized as

$$
C(T)=C_{0}\left(\left[1+\delta_{c}\right]-\frac{T_{C}}{T}\right)^{\gamma_{c}}
$$

For two light quarks and one heavy quark, the parameters take the values $C_{0}=1.16, \delta_{c}=0.02$ and $\gamma_{c}=0.29$ (see figure 1) an integration constant that is fixed by Gibbs' condition $p_{Q G P}=p_{\text {hadr }}$ at $T_{C}$.

Figure 2 shows the pressure, energy and entropy density for two light quark flavours $\left(m_{u, d}=0\right)$ and a heavier strange quark $\left(m_{s} \simeq 170 \mathrm{MeV}\right)$ in our confinement model.

We now proceed as follows: We adopt the picture of the QGP as a quasiparticle gas consisting of massive 'quarks' and 'gluons' which are the relevant thermodynamical degrees of freedom. The resulting EoS serves as input for the construction of the fireball which is discussed in section 5 . Furthermore, within this framework the quasiparticle $q \bar{q}$-loop becomes the only contribution to the photon self-energy in eq.(11) since the quasiparticles are by construction non-interacting. The reduced probability for thermally exciting a quark because of confinement, and the thermal effective masses, have to be taken into account properly as discussed in the next section.

\section{Calculation of the photon spectral function}

\footnotetext{
${ }^{\dagger}$ We mention that the proposed method relies on input from the lattice. Whereas high precision data exist in the pure gauge sector, calculations with dynamical quarks are not yet in a satisfactory position to yield proper continuumextrapolated results with physical quark masses. We estimate that the results obtained within our confinement model may still change by $5-15 \%$ in the vicinity of $T_{C}$ once high statistics data are available. However, this small correction does not influence the results of the following discussion.
} 


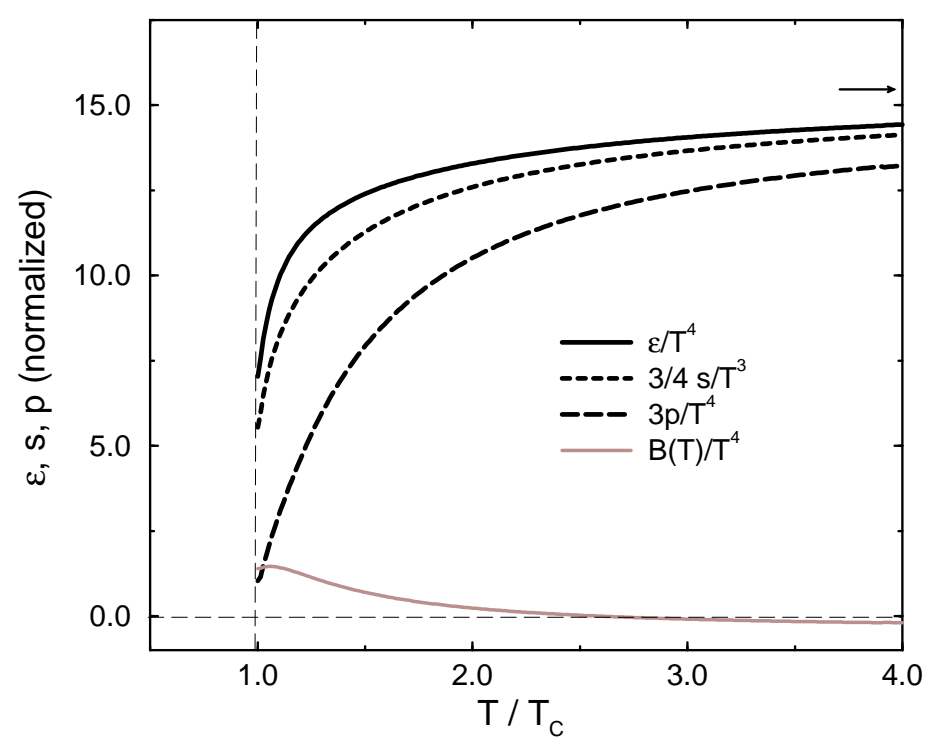

Figure 2: Pressure, energy density and entropy density for two light quark flavours $\left(m_{u, d}=0\right)$ and a heavier strange quark $\left(m_{s} \simeq 170 \mathrm{MeV}\right)$ in our quasiparticle model. The arrow indicates the ideal gas limit of massless three-flavour QCD. The function $B(T)$ is also shown.

\subsection{The quark-gluon phase}

As long as the thermodynamically active degrees of freedom are quarks and gluons, the timelike photon couples to the continuum of thermally excited $q \bar{q}$ states and subsequently converts into a charged lepton pair. The calculation of the photon spectral function at the one-loop level is performed using standard thermal field theory methods. The well-known leading-order result for bare quarks and gluons as degrees of freedom is:

$$
\begin{aligned}
\operatorname{Im} \bar{\Pi}\left(q^{0}, \mathbf{q}, T\right) & =-\frac{q^{2}}{12 \pi} \cdot 3 \sum_{f=u, d, s} \theta\left(q^{2}-4 m_{f}^{2}\right) e_{f}^{2}\left(1+\frac{2 m_{f}^{2}}{q^{2}}\right) \sqrt{1-\frac{4 m_{f}^{2}}{q^{2}}} \\
\times & \left(1+2\left[\frac{T}{|\mathbf{q}|} \frac{1}{\sqrt{1-\frac{4 m_{f}^{2}}{q^{2}}}} \ln \left(\frac{f_{D}\left(\frac{q_{0}}{2}-\frac{|\mathbf{q}|}{2} \sqrt{1-\frac{4 m_{f}^{2}}{q^{2}}}\right)}{f_{D}\left(\frac{q_{0}}{2}+\frac{|\mathbf{q}|}{2} \sqrt{1-\frac{4 m_{f}^{2}}{q^{2}}}\right)}\right)-1\right]\right),
\end{aligned}
$$

where $q=\left(q^{0}, \mathbf{q}\right)$ is the four-momentum of the virtual photon, $e_{f}$ the quark electric charge and $m_{f}$ the quark mass of flavour $f$. This result, however, holds only up to perturbative higher order corrections in $g_{s}$ that take into account collective plasma effects. Here, contributions from soft gluons lead to strong modifications. The corresponding two- and three-loop contributions show no clear convergence 17. 18. Close to the phase transition, we also expect non-perturbative confinement physics to enter. Consequently, we follow a different approach.

Recalling the results of the previous section, the thermodynamic properties of the QGP as given by lattice QCD are well reproduced by a gas of quasiparticles. Let us now assume that a quark quasiparticle couples to a photon in the same way as a bare quark (a form factor representing the 'cloud' of 
the quasiparticle could in principle also be included, but in absence of information about the detailed quasiparticle structure we ignore this point). For a gas of non-interacting quasiparticles, the one-loop result for $\operatorname{Im} \bar{\Pi}$ is adequate, with input properly adjusted. All higher order QCD effects manifest in the thermal quasiparticle masses $m(T)$, the function $B(T)$ and the confinement factor $C(T)$. Incorporation of the first two features in the calculation is straightforward. The bare quark masses in eq. (12) simply have to be replaced by the $T$-dependent quasiparticle masses for each flavour, see eq. (7). The thermal vacuum energy $B(T)$ does not contribute to the dilepton rate.

The naive replacement $f_{D} \rightarrow C(T) f_{D}$ is, however, not permitted in eq. (12). Since any modification of the free particle distribution functions leads to non-equilibrium field theory, products of delta functions (pinch singularities) may arise in loop calculations. Therefore, the quasiparticle model as it stands cannot be used in expressions derived from simple perturbative thermal field theory. Recalling the physical interpretation of the confinement factor $C(T)$, we can use the expression for the dilepton rate, eq. (1), instead. The mechanism for dilepton production at tree-level is the annihilation of a $q \bar{q}$ pair into a virtual photon where the quark lines are multiplied by the distributions $f_{D}(T)$, giving the probability of finding a quark or an antiquark in the hot medium. This also becomes clear when we look at the limit $\mathbf{q} \rightarrow 0$ of eq. (12). Then,

$$
\operatorname{Im} \bar{\Pi}\left(q^{0}, T\right) \sim \operatorname{Im} \bar{\Pi}\left(q^{0}, T=0\right) \cdot\left(1-2 f_{D}\left(q^{0} / 2\right)\right),
$$

and the temperature enters only in the Pauli-blocking of the quarks propagating in the loop. Now, from eq. (11)

$$
\frac{d N}{d^{4} x d^{4} q} \sim f_{B}\left(q^{0}\right) \operatorname{Im} \bar{\Pi}(q, T) .
$$

Combining the different thermal occupation factors, we end up with the well-known result

$$
\frac{d N}{d^{4} x d^{4} q} \sim\left[f_{D}\left(q^{0} / 2\right)\right]^{2}
$$

so the differential dilepton rate is proportional to the probability of finding a quark $q$ times the probability of finding an antiquark $\bar{q}$ with the correct momentum, as anticipated The incorporation of the confinement factor is now obvious: since it reduces the number of thermally active degrees of freedom, it also reduces the dilepton rate by a factor of $C(T)^{2}$.

In summary, eq. (1) can be used to calculate the dilepton rate originating from a hot QGP phase, provided an overall factor $C(T)^{2}$ is applied to account for the reduced probabilities, and the bare masses $m_{f}$ in the one-loop expression (12) are replaced by the $T$-dependent thermal masses (7). The rôle of the factor $C(T)$ is illustrated in figure 3, where the differential dilepton rate originating from a hot QGP in the quasiparticle approach is shown for different temperatures. Note that the plotted quantity is independent of the fireball volume, so the resulting differences are only due to the dropping quasiparticle masses and the squared confinement factor $C(T)$, which is responsible for a decrease by more than an order of magnitude at $T \sim T_{C}$ as compared to the highest temperature shown. One also observes that, as expected, the (negative) slope of the production rate in the region of high invariant mass gets steeper as the temperature decreases. It is important to note that this setup neglects contributions from hadronic degrees of freedom above $T_{C}$. As mentioned, quarks and gluons become clustered into hadrons (glueballs, mesons) as the temperature approaches $T_{C}$ from above. These hadronic excitations are comparatively heavy and thus do not contribute much to the thermodynamics. Since we do not know in detail how the statistical re-arrangement of degrees of freedom occurs, we refrain from including these hadronic sources of dilepton yield above $T_{C}$. Our calculation is therefore expected to give a lower limit on the leptonic radiation from the QGP phase.

\footnotetext{
¥) We neglect a possible chemical potential for the quarks. For a finite $\mu$, the corresponding expression would be $\frac{d N}{d^{4} x d^{4} q} \sim f_{D}\left(\left(q^{0}-\mu\right) / 2\right) f_{D}\left(\left(q^{0}+\mu\right) / 2\right)$.
} 


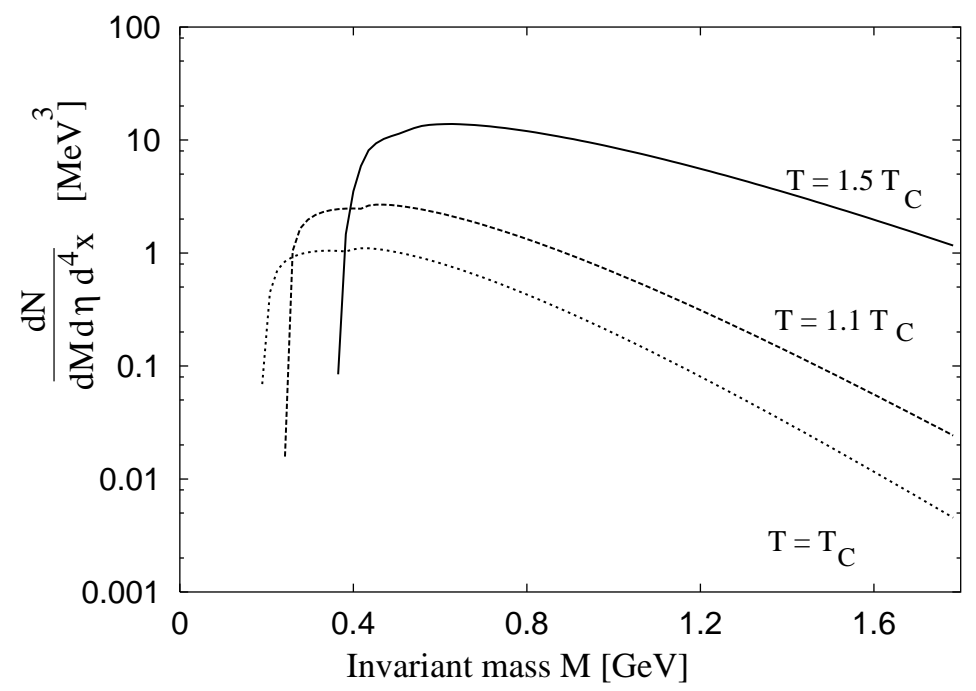

Figure 3: Dilepton rates originating from the QGP phase in the quasiparticle model for different temperatures.

The quasiparticle model does not take into account the collective plasma modes, such as the (longitudinal) gluonic plasmons and the (helicity-flipped) quark plasminos. Since their residues are exponentially suppressed in the HTL approximation for thermal momenta $k \sim T$, their contributions to the thermodynamical quantities are negligible. However, in the case of soft dilepton production it is well known that these modes lead to sharp, distinct structures in the spectrum, referred to as Van Hove singularities [23. The plasmino branch has a minimum in its dispersion relation at $k \neq 0$ (which follows on very general grounds [24], independent of the HTL approximation). This leads to a diverging density of states which, in turn, shows up in the dilepton spectrum as a pronounced peak. Our model cannot exhibit, by construction, such plasmino effects. However, since the peaks are roughly located at $\simeq 2 m_{q}(T)$, where $m_{q}(T)$ is the thermal mass of the quasiparticles, these Van Hove singularities would be smeared out by the fireball evolution. The thermal mass drops as the temperature goes down, dragging along the peak position with it. Furthermore, since $m_{q}(T)$ is of the order of the temperature $T$ or smaller in our model, the singularities appear at low invariant mass $(<500 \mathrm{MeV})$ where they are overwhelmed by the hadronic part of the dilepton production. Therefore the presence of these collective modes would presumably not influence our results for the dilepton rate in the QGP phase.

Our model of the QGP phase also finds preliminary support from a recent lattice calculation [51] of thermal vector meson correlation functions above $T_{C}$ in quenched QCD with Clover improved Wilson fermions. Using the maximum entropy method, the vector spectral function was extracted from the corresponding current correlator. Although the statistical uncertainties are still considerable, it is interesting to note that the resulting spectrum resembles the free spectral function, as in our case, and has a gap at low energies given by a thermal mass threshold of $(2-3) T$, which is indeed close to $2 m_{q}(T)$, the natural cut-off of the spectrum and, correspondingly, of the thermal dilepton radiation in the confinement model (see figure 3). Furthermore, this result seems to rule out heavy quark quasiparticles in the deconfined phase, as predicted by other phenomenological models [20, 21]. Of course, higher statistics and improved actions are mandatory to confirm these observations. 


\subsection{The hadronic phase}

Below $T_{C}$, confinement sets in and the effective degrees of freedom change to colour singlet, bound $q \bar{q}$ or $q q q(\bar{q} \bar{q} \bar{q})$ states. The photon couples now to the lowest-lying 'dipole' excitations of the vacuum, the hadronic $J^{P}=1^{-}$states: the $\rho, \omega$ and $\phi$ mesons and multi-pion states carrying the same quantum numbers. The electromagnetic current-current correlation function can be connected to the currents generated by these mesons using an effective Lagrangian which approximates the $S U(3)$ flavour sector of QCD at low energies. The appropriate model for our purposes is the improved Vector Meson Dominance model combined with chiral dynamics of pions and kaons as described in [25. Within this model, the following relation between the imaginary part of the irreducible photon self-energy $\operatorname{Im} \bar{\Pi}$ and the vector meson self-energies $\Pi_{V}(q)$ in vacuum is derived:

$$
\operatorname{Im} \bar{\Pi}(q)=\sum_{V} \frac{\operatorname{Im} \Pi_{V}(q)}{g_{V}^{2}}\left|F_{V}(q)\right|^{2}, \quad F_{V}(q)=\frac{\left(1-\frac{g}{g_{V}^{0}}\right) q^{2}-m_{V}^{2}}{q^{2}-m_{V}^{2}+i \operatorname{Im} \Pi_{V}(q)},
$$

where $m_{V}$ are the (renormalized) vector meson masses, $g_{V}^{0}$ is the $\gamma V$ coupling and $g$ is the vector meson coupling to the pseudoscalar Goldstone bosons $\pi^{ \pm}, \pi^{0}$ and $K^{ \pm}, K^{0}$. Eq.(16) is valid for a virtual photon with vanishing three-momentum q. For finite three-momenta there exist two scalar functions $\bar{\Pi}_{L}$ and $\bar{\Pi}_{T}$, because the existence of a preferred frame of reference (the heat bath) breaks Lorentz invariance, and one has to properly average over them. However, taking the limit $|\mathbf{q}| \rightarrow 0$ should be reasonable for our purposes in view of the fact that the c.m. rapidity interval accessible at CERES and RHIC restricts $|\mathbf{q}|$ on average to only a fraction of the vector meson mass $m_{V}$.

Finite temperature modifications of the vector meson self-energies appearing in eq.(16) are calculated using thermal Feynman rules. The explicit calculations for the $\rho$ - and $\phi$-meson can be found in ref.[10]. At the one-loop level, the $\rho$ and $\phi$ are only marginally affected by temperature even close to $T_{C}$ because of the comparably large pion and kaon masses: $m_{\pi} \simeq T_{C}, m_{K} \simeq 3 T_{C}$. The thermal spectral function of the $\omega$-meson has been discussed in detail in [26]. Here, the reaction $\omega \pi \rightarrow \pi \pi$ was found to cause a considerable broadening of the $\omega$ spectral function, leading to $\Gamma_{\omega}\left(T_{C}\right) \simeq 7 \Gamma_{\omega}(0)$. The corresponding photon spectral function is displayed in figure (left panel).

At higher invariant masses $1 \mathrm{GeV}<M<2 \mathrm{GeV}, \pi a_{1}$ annihilation is the dominant source of dileptons [27, 28]. The vacuum vector and axialvector spectral functions become mixed to order $T^{2}$ with a strength $T^{2} /\left(6 f_{\pi}^{2}\right)\left(f_{\pi} \simeq 93 \mathrm{MeV}\right.$ is the pion decay constant) due to their coupling to the pionic heat bath [29] and should be degenerate at the point of chiral symmetry restoration. The effect of the $a_{1}$ and higher resonances can then be approximately subsumed in a structureless continuum above $1 \mathrm{GeV}$ 30]. We practically implement the $\pi a_{1}$ contribution by adding a flat $2 \pi$ continuum to the resonance $\rho$ meson spectral function that feeds into the photon spectral function, reminiscent of the perturbative plateau of $q \bar{q}$ annihilation.

There is still considerable stopping of the interpenetrating nuclei at SPS energies, resulting in a net baryon density $\rho_{B}$ in the central rapidity region. At RHIC, measurements [31, 32] indicate that the proton over antiproton excess is small, implying that the baryons are distributed over a larger rapidity interval. Therefore, finite baryon density effects should not play such an important role at RHIC kinematics.

For the evaluation of density effects which are relevant at SPS conditions, we use the results discussed in [33]. There it was shown that in the linear density approximation, $\Pi_{V}$ is related to the vector meson - nucleon scattering amplitude $T_{V N}$ :

$$
\Pi_{V}\left(q^{0}, \vec{q}=0 ; \rho\right)=\Pi_{V}^{v a c}-\rho_{B} T_{V N}(q), \quad T_{V N}(q)=-\frac{i}{3} \int d^{4} x e^{i q x}\left\langle N\left|\mathcal{T} j_{\mu}(x) j^{\mu}(0)\right| N\right\rangle,
$$

with $|N\rangle$ being zero-momentum free nucleon states. In the following, we assume that the temperatureand density dependences of $\Pi_{V}$ factorize, i.e. we replace $\Pi_{V}^{v a c}$ in eq.(17) by the temperature-dependent 

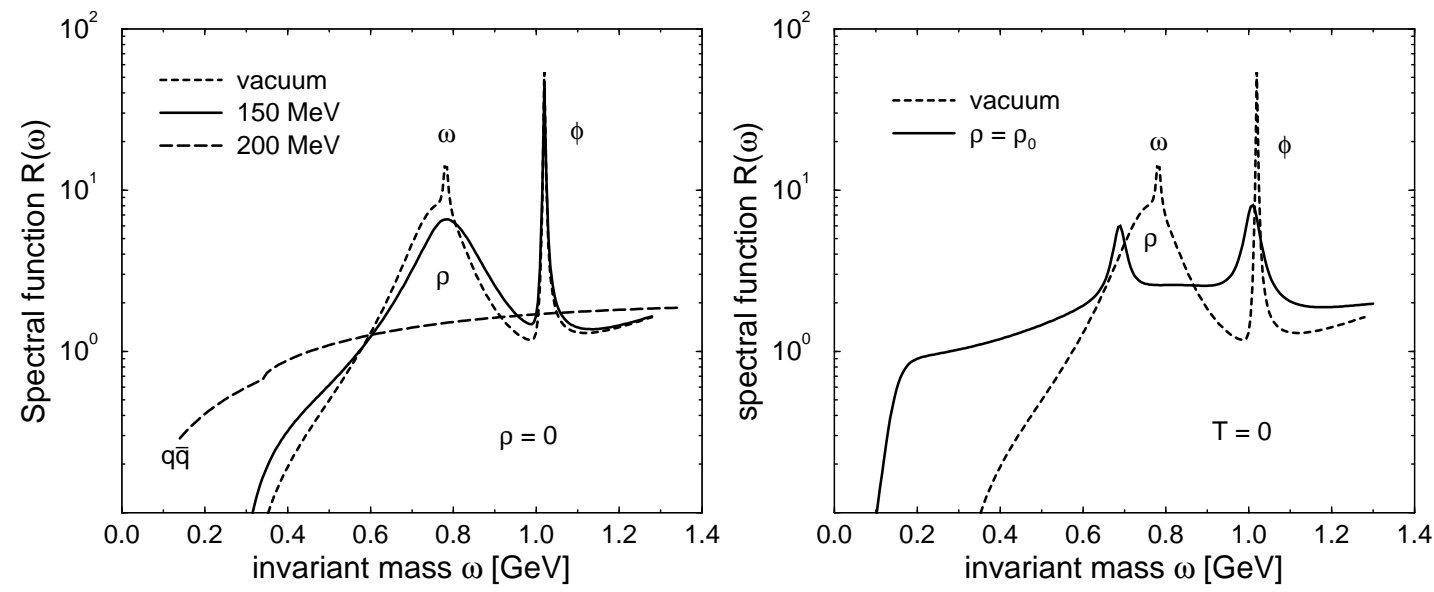

Figure 4: The photon spectral function $R(\omega)=\left(12 \pi / \omega^{2}\right) \operatorname{Im} \bar{\Pi}(\omega)$ at finite temperature and $\rho_{B}=0$ (left panel) and at $T=0$ and baryon density of normal nuclear matter, $\rho_{B}=\rho_{0}=0.17 \mathrm{fm}^{-3}$ (right panel). For orientation, the $q \bar{q}$ line in the left panel shows the spectral function in the QGP phase with massless $u$ - and $d$-quarks and $m_{s}=150 \mathrm{MeV}$ for $s$-quarks, neglecting $\alpha_{s}$-corrections.

$\Pi_{V}(T)$ and leave $T_{V N}$ unaffected. This amounts to neglecting contributions from matrix elements such as $\left\langle\pi N\left|\mathcal{T} j_{\mu}(x) j^{\mu}(0)\right| \pi N\right\rangle$ (nucleon-pion scatterings where the pion comes from the heat bath). Furthermore, this approximation does not take into consideration a possible $T$-dependent pion or nucleon mass. Some effective models suggest that, near the phase transition, the nucleon mass follows the behaviour of the chiral condensate $\langle\bar{\psi} \psi\rangle$ and drops abruptly as the quarks lose their constituent masses. Such modifications of particle properties may have a considerable impact on the spectral functions. However, since the temperature range over which the dropping takes place is narrow, we expect such effects not to leave distinct signals in the dilepton spectra which are only sensitive to the integrated time (and hence temperature) evolution of the system.

The photon spectral function at finite density and zero temperature is depicted in figure 4 (right panel). The interaction with nucleons causes a strong broadening of the $\rho$ meson down to the one pion threshold, leading to a complete dissolution of its quasiparticle peak structure. The modifications of the $\omega$ and $\phi$ meson spectral distributions are more moderate: The mass of the $\omega$ drops by about $100 \mathrm{MeV}$ at normal nuclear matter density, and its width increases by a factor of about 5 , whereas the $\phi$ mass stays close to its vacuum value, accompanied by a ninefold increased width.

To summarize, the most prominent changes of the photon spectral function, when compared to the vacuum case, arise from the broadening of the $\rho$ due to finite baryon density effects and the broadening of the $\omega$ due to scattering off thermal pions. The $\phi$ meson retains its distinct peak structure even under extreme conditions of density and temperature. Very close to $T_{C}$, however, these results based on perturbative calculations, are not expected to be reliable.

\subsection{After freeze-out contributions}

At the freeze-out stage, there are still vector mesons present. These will decay with their vacuum properties on their way to the detector and add to the dilepton yield from the previous thermalized phase. The invariant mass region below approximately $400 \mathrm{MeV}$ is mainly filled by the Dalitz decays of the vector mesons. We take these contributions from the experimental analysis of the CERES collaboration for SPS conditions. Since the PHENIX acceptance starts only above $1 \mathrm{GeV}$, the Dalitz decays do not 
play a significant rôle at RHIC.

For the calculation of the direct decay of a vector meson $V$ into a lepton pair we start with the following formula:

$$
\frac{d N_{V}}{d M d \eta}=\frac{1}{\Delta \eta} \frac{\alpha^{2}}{12 \pi^{4}} R_{V}(M, T=0) \int_{\tau_{f}}^{\infty} d \tau V_{f} \int d^{3} q \frac{M}{q^{0}} f_{B}\left(q^{0}, T_{f}\right) \exp \left(-\frac{\tau-\tau_{f}}{\gamma(q) \tau_{0}^{V}}\right) .
$$

Here, $T_{f}$ and $V_{f}$ are the fireball temperature and volume, respectively, at freeze-out. After $\tau_{f}$, the freezeout time, all medium effects are switched off, so the vacuum photon spectral function $R_{V}(M, T=0)$ determines the rate. The corresponding momentum distribution is given by the thermal Bose function, evaluated at the freeze-out temperature $T_{f}$. However, the absolute number $N$ of vector mesons as a function of time is not a constant: since the mesons decay and there is no thermal recombination, $N$ decreases exponentially like $\exp \left(-\left(\tau-\tau_{f}\right) /\left(\gamma(q) \tau_{0}^{V}\right)\right)$. The vacuum life time of the vector meson $V$ under consideration is denoted by $\tau_{0}^{V}$, and $\gamma(q)$ accounts for time dilatation effects on particles with finite three-momentum:

$$
\gamma(q)=\frac{1}{\sqrt{1-v^{2}}}=\frac{q^{0}}{M}
$$

After the time integration we end up with:

$$
\frac{d N}{d M d \eta}=\frac{1}{\Delta \eta} \frac{\alpha^{2}}{12 \pi^{4}} \tau_{0} R(M, T=0) V_{f} \int d^{3} q f_{B}\left(q^{0}, T_{f}\right) .
$$

The averaged space-time four-volume that is available after freeze-out is therefore $V_{f} \tau_{0}^{V}$, as anticipated. The integral over $d^{3} q$ now yields the freeze-out particle density $n(M)=N(M) / V$ of the virtual photons as a function of invariant mass. Note that the information on the vector mesons remains entirely in the photon spectral function. With the factor $V_{f}$ we obtain the total number of photons at freeze-out. When weighted with $R_{V}(M)$, this gives the $d N / d M$ distribution for the process meson $\rightarrow \gamma^{*} \rightarrow e^{+} e^{-}$.

We have also checked that a commonly used Breit-Wigner ansatz of the form

$$
\frac{d N_{e^{+} e^{-}}}{d M}=\left[V_{f} n\left(T_{f}, M\right)\right] \xi B_{V} \frac{M^{2} \Gamma_{V}(M)}{\left(M^{2}-m_{V}^{2}\right)^{2}+M^{2} \Gamma_{V}(M)^{2}}
$$

yields almost identical results. Here, $\Gamma_{V}(M)$ stands for the $M$-dependent decay width, $\xi$ is the normalization factor and $B_{V}$ the branching ratio for the leptonic decay of the corresponding vector meson.

\subsection{Drell-Yan and charm contributions}

At invariant masses $M>1 \mathrm{GeV}$ the Drell-Yan mechanism, i.e., hard quark-antiquark annihilation $q \bar{q} \rightarrow \ell^{+} \ell^{-}$at leading order (LO), constitutes another source of (non-thermal) dileptons. Its differential $\mathrm{LO}$ cross section in a nucleus $\left(A_{1}\right)$-nucleus $\left(A_{2}\right)$ collision reads

$$
\begin{aligned}
& \frac{d \sigma\left(A_{1} A_{2}\right)}{d y d M}=\frac{8 \pi \alpha^{2}}{9 M s} \sum_{q} e_{q}^{2} \times \\
& {\left[\left(Z_{1} f_{q}^{p}\left(x_{1}\right)+\left(A_{1}-Z_{1}\right) f_{q}^{n}\left(x_{1}\right)\right)\left(Z_{2} f_{\bar{q}}^{p}\left(x_{2}\right)+\left(A_{2}-Z_{2}\right) f_{\bar{q}}^{n}\left(x_{2}\right)\right)+(q \leftrightarrow \bar{q})\right]}
\end{aligned}
$$

where $\sqrt{s}$ denotes the c.m. energy of the nucleon-nucleon collision and $x_{1,2}=M / \sqrt{s} \exp ( \pm y)$ is the momentum fraction of the beam and target parton respectively.

The Drell-Yan cross section (19) is computed using the LO MRST parameterization [34] for the parton distributions $f_{i}^{p}\left(x, \mu^{2}\right)$ evaluated at the hard scale $\mu^{2}=M^{2}$. However, it has been checked that using 
different LO sets (e.g., CTEQ5L [35] or GRV98LO [36]) affects the results by only $10 \%$ at SPS and $20 \%$ at RHIC energies. To account for higher order corrections, we multiply the LO expression (19) by a $K$ factor $K=2$ fitted from $p-p$ data [37. Finally, nuclear effects like shadowing or quark energy loss are expected to suppress the Drell-Yan yield by about 30-50\% [38, 39]. Since these effects are still poorly known quantitatively, we neglect them and consider our Drell-Yan rate as an upper limit on the actual rate.

Using the Glauber model of multiple independent collisions, the average dilepton multiplicity in a $A_{1}-A_{2}$ collision at impact parameter $b$ is given by

$$
\frac{d N\left(A_{1} A_{2}\right)}{d y d M}(b)=T_{A_{1} A_{2}}(b) \times K \frac{d \sigma\left(A_{1} A_{2}\right)}{d y d M},
$$

where the normalized thickness function $T_{A_{1} A_{2}}(b)$ is computed assuming the standard Woods-Saxon nuclear density profile. The Drell-Yan pair multiplicity (20) is then averaged for the $30 \%$ and $6 \%$ most central collisions to be compared with CERES and PHENIX data, respectivelyß).

Another source of dileptons in the high invariant mass region consists of semileptonic decays of charmed mesons. Whereas earlier calculations found a considerable yield from open charm exceeding the thermal radiation [40], the subsequent inclusion of medium effects like energy loss led to a suppression of the dielectron rate and made it comparable to or even lower than the Drell-Yan yield [42, 41. Since the Drell-Yan contribution plays only a subdominant rôle in the following, we will not explicitly include the open charm contributions.

\section{$5 \quad$ Fireball model}

\subsection{General properties}

We do not aim at the detailed description of the heavy-ion collision or its subsequent expansion on an event by event basis or by a hydrodynamical simulation. Instead we use a model of an expanding fireball which enables us to test different scenarios with different parameter sets in a systematic way, so as to gain insight into the time evolution of the strongly interacting system.

We assume that the physics of the fireball is the same inside each volume of given proper time $\tau$, thus averaging over spacial inhomogenities in density and temperature. The volume itself is taken to be an expanding cylinder, in which the volume elements move away from the center in order to generate the observed flow. There is no global Lorentz frame in which thermodynamics can be applied. As the fireball expands, volume elements away from the center are moving with large velocities and are subject to time dilatation when seen in the center of mass frame of the collision. We assume a linear rise in rapidity when going from central volume elements to the fireball edge along the beam $(z)$-axis and the transverse axis. As the velocities along the $z$-axis are typically large (up to $c$ ) as compared to transverse motion (up to $0.55 \mathrm{c}$ ) for SPS and RHIC conditions, we make the simplifying assumption that the proper time is in a one-to-one correspondence to the $z$-position of a given volume element, thus neglecting the time dilatation caused by transverse motion. The whole system is assumed to be in local thermal (though not necessarily in chemical) equilibrium at all times.

Given this overall framework, the volume expansion of the fireball is governed by the longitudinal growth speed $v_{z}$ and the transverse expansion speed $v_{\perp}$ at a given proper time. These quantities can be

\footnotetext{
§) In addition to these centrality cuts, we need to rely on further assumptions to take properly into account the acceptance of these experiments. Therefore, we shall assume the generic form $d \sigma / d p_{\perp} \propto p_{\perp} /\left(1+\left(p_{\perp} / p_{0}\right)^{2}\right)^{6}\left(p_{0}=3 \mathrm{GeV}\right)$ for the $p_{\perp}$ dependence of the DY process [37]. Furthermore, neglecting corrections due to the intrinsic $k_{\perp}$ of the incoming partons, the angular distribution is taken to be $d N / d \Omega \propto 1+\cos ^{2} \theta$ where $\theta$ is the angle between the lepton and the beam axis.
} 
determined at the freeze-out point and correspond to the observed amount of flow. However, flow is measured in the lab frame and needs to be translated into the growth of proper time volume. We use a detailed analysis of the freeze-out conditions for central $\mathrm{Pb}-\mathrm{Pb}$ collisions at $160 \mathrm{AGeV}$ [45] to fix the endpoint of the evolution. The initial state is constrained using the overlap geometry of the colliding nuclei. The expansion between initial and freeze-out stages is then required to be in accordance with the EoS as determined from the quasiparticle model described in section 3. The resulting model serves as the basic setup, its extension to different beam energies and collision centralities is discussed in 5.5 .

\subsection{Initial and Freeze-out conditions}

We use the data set b1 of 45 as the endpoint of our fireball evolution. The data set has been obtained by a simultaneous fit of the fireball emission function to hadronic $m_{T}$ spectra and HBT radii, so as to disentangle the contributions from flow and temperature to these quantities.

The fireball is characterized by a transverse box-shaped density distribution with a radius $R_{B}=12.1 \mathrm{fm}$. This corresponds to a root mean square radius of $R_{r m s}^{f}=8.55 \mathrm{fm}\left(=R_{B} / \sqrt{2}\right)$. The average transverse expansion velocity is found to be $0.5 c$ and the temperature at freeze-out is $T_{f}=100 \mathrm{MeV}$.

Looking at the longitudinal expansion, a velocity $v_{z}^{f} \approx 0.9 c$ at the fireball front is needed in $\pm z$ direction in order to account for the observed shape of $d N_{c h} / d y$ distributions.

The initial conditions in transverse direction can be fixed by the overlap geometry. Here, $R_{0} \approx 4.5 \mathrm{fm}$ and $v_{\perp}=0$ for central collisions. The initial longitudinal size of the fireball is related to the amount of stopping of the matter in the collision and the time $\tau_{0}$ necessary for the formation of a thermalized system. There is no direct information on these two quantities. However, pQCD calculations indicate $\tau_{0} \approx 1 \mathrm{fm} / c$ for SPS conditions and shorter times at RHIC. The initial longitudinal velocity $v_{z}^{i}$ can be inferred from hydrodynamical calculations. Since the initial state in principle determines the final state (if the EoS is known), one can fit this parameter to the observed $d N / d y$ spectra. This procedure points at $v_{z}^{i} \approx 0.5 \mathrm{c}$. The initial longitudinal size of the system at proper time $\tau_{0}$ is then calculated by the intercept of $z(t)=v_{z}^{i} \cdot t$ with the $\tau=\sqrt{t^{2}-z^{2}}=\tau_{0}$ line.

\subsection{Volume evolution}

Using the available information on initial and freeze-out conditions and the EoS of the system, we are now able to reconstruct the evolution of the fireball volume in proper time:

(1) The EoS translates into a temperature (and hence $\tau$ ) dependent acceleration profile $a=$ const. . $p(T) / \epsilon(T)$ that exhibits a soft transition point at $T=T_{C}$. This can be understood as follows: the thermal vacuum energy $B(T)$ adds to the energy density $\epsilon$ but is subtracted from the pressure $p$. As evident from figure 2, $B(T)$ gets large near the phase transition and correspondingly the ratio $p / \epsilon$ drops. In the interpretation of $B(T)$ as a 'bag pressure', the pressure of the QGP becomes partly counterbalanced by the confining bag pressure. Comparing initial and final conditions, we need both longitudinal and transverse acceleration to account for the velocity gain, and we keep the possibility of having two different constants $c_{\perp}$ and $c_{z}$ which relate transverse and longitudinal accelerations to $p / \epsilon$. In practice, the temperature evolution with $\tau$ is calculated starting with a trial ansatz and iterating the result to obtain a self-consistent solution.

(2) Starting with an ansatz for the radial expansion velocity,

$$
v_{\perp}(\tau)=\int_{0}^{\tau} d \tau^{\prime} c_{\perp} \frac{p\left(\tau^{\prime}\right)}{\epsilon\left(\tau^{\prime}\right)}
$$


and

$$
R(\tau)=R_{0}+\int_{0}^{\tau} \int_{0}^{\tau^{\prime}} d \tau^{\prime} d \tau^{\prime \prime} c_{\perp} \frac{p\left(\tau^{\prime \prime}\right)}{\epsilon\left(\tau^{\prime \prime}\right)}
$$

where $R_{0}$ is the initial overlap rms radius of the collision region, we fix the two unknown parameters $c_{\perp}$ and $\tau_{f}$ by the requirement that $R\left(\tau_{f}\right)=R_{r m s}^{f}$ and $v_{\perp}\left(\tau_{f}\right)=v_{\perp}^{f}$.

(3) For the longitudinal expansion we follow the motion of the fireball front in the center of mass frame and use the expressions

$$
v_{z}(t)=v_{z}^{i}+\int_{0}^{t} d t^{\prime} c_{z} \frac{p\left(t^{\prime}\right)}{\epsilon\left(t^{\prime}\right)}
$$

and

$$
z(t)=z_{0}+v_{z}^{i} \cdot t+\int_{0}^{t} \int_{0}^{t^{\prime}} d t^{\prime} d t^{\prime \prime} c_{z} \frac{p\left(t^{\prime \prime}\right)}{\epsilon\left(t^{\prime \prime}\right)} .
$$

The parameters $c_{z}$ and $t_{f}$ (freeze-out in c.m. frame) are fixed by $v_{z}\left(t_{f}\right)=v_{z}^{f}$ and $z\left(t_{f}\right)$ to lie on the $\tau=\tau_{f}$ line, with $\tau_{f}$ determined from the radial expansion.

(4) The movement of the fireball front in the c.m. frame can now be translated into the growth of volumes in proper time by finding the intercept of $z(t)$ and $\tau=$ const. and calculating the pathlength along this curve of fixed proper time.

In order to construct a self consistent evolution model, we need to find the proper ratio $p(T(\tau)) / \epsilon(T(\tau))$ corresponding to the volume expansion. The evolution of $T(\tau)$ is dealt within the next section. For the QGP phase, the ratio is then determined within the quasiparticle model. Unfortunately, no reliable information on $p / \epsilon$ is available for the hadronic phase. We can, however, deduce the value of this ratio at both $T_{C}$ and $T_{f}$, and interpolate between these limits. As the lattice indicates neither a sharp drop in $\epsilon$ nor in $p$ when approaching $T_{C}$ from above, it appears reasonable to assume that the $p / \epsilon$ ratio stays small in the vicinity of the phase transition even in the hadronic phase. On the other hand, the decoupling of the system at freeze-out implies that interactions between its constituents become unimportant, therefore we recover the ideal gas limit at $T \rightarrow T_{f}$, where standard thermodynamics predicts $p / \epsilon=1 / 3$ (for massless particles). We now interpolate linearly between these two values to cover the temperature region inbetween.

\subsection{Temperature evolution}

The temperature profile $T(t)$ of the fireball is uniquely determined by the assumed condition of isentropic expansion once the volume expansion is known. In order to derive the temperature $T$ at a given time, we calculate the entropy density via

$$
s(\tau)=S_{0} / V(\tau),
$$

where $S_{0}$ is the total entropy of the fireball. The relation between the entropy density $s$ and the fireball temperature $T$ is fixed by the EoS. By inverting this (unique) relation numerically, we finally obtain the temperature profile.

We infer the EoS of the QGP phase from the thermal quasiparticle model [19] adapted to lattice QCD results. Unfortunately, determining the behaviour of the hadronic phase is not quite as easy for a number of reasons. First, lattice results usually employ quark masses which are far too large, leading to 
pion masses $m_{\pi}^{\text {latt }} \gtrsim 3 m_{\pi}^{\text {phys }}$. The resulting thermal suppression of these degrees of freedom causes a considerable discrepancy of the lattice EoS with respect to that of the interacting pion gas or the free hadronic resonance gas. On the other hand, the description in terms of a non-interacting or perturbatively interacting system is bound to fail near the phase transition. Only in the very final stages, near the freeze-out, interactions cease to be important and one may assume that a non-interacting system describes the situation adequately.

We parametrize our insufficient knowledge close to $T_{C}$ by interpolating smoothly between two regimes, the QGP quasiparticle result for $T>T_{C}$ and the noninteracting hadronic resonance gas result for $T<T_{f}$. This approach is supported by the general shape of the EoS on the lattice for two light quarks and one heavy quark, which resembles a weak first-order or second order phase transition, or even a smooth crossover [3].

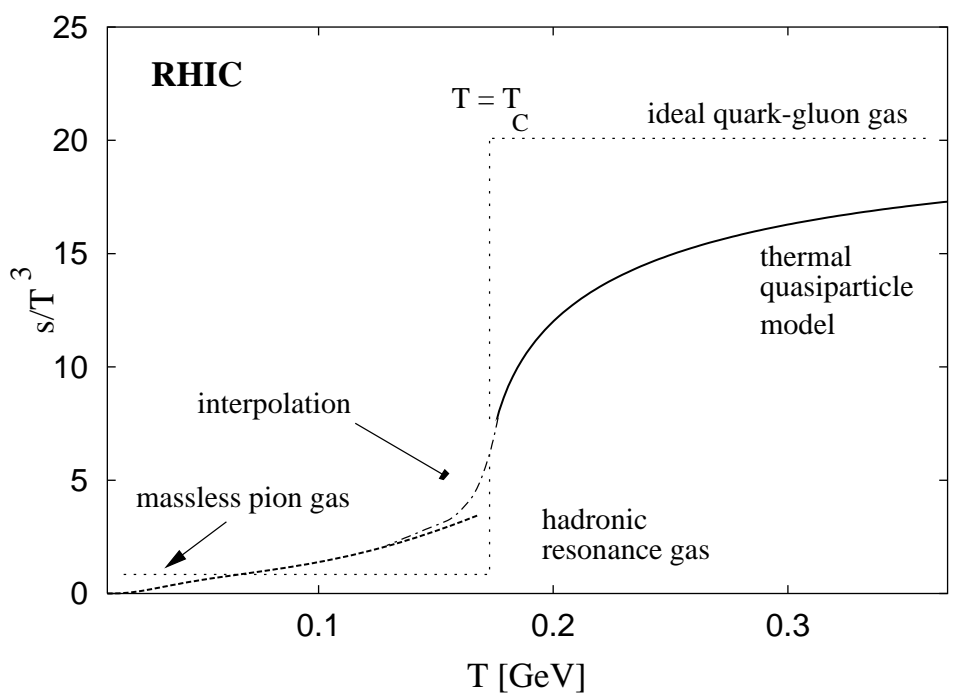

Figure 5: The temperature dependence of the entropy density $s(T)$ in the RHIC kinematical scenario as compared to the ideal quark-gluon gas and the massless noninteracting pion gas limit (dotted line). The three relevant regions used in the model calculation are given as ideal hadronic resonance gas (solid line), interpolation (dash-dotted line) and thermal quasiparticle model (dashed line).

The situation is shown for the RHIC scenario in figure 5 and compared to the ideal quark-gluon gas and the hadronic resonance gas approach used in other works (e.g. [6, 7]). One can clearly observe that the deviations in both the quark-gluon phase and the hadronic phase from the ideal gas are large, amounting to a factor of more than two for the entropy density near $T_{C}$. Keeping in mind that a smaller entropy density translates into a higher temperature in an isentropic expansion, we conclude that our model predicts a prolonged lifetime of the QGP phase as compared to the ideal gas ansatz, whereas the lifetime of the hadronic phase is reduced somewhat.

At SPS, the measured ratio of protons over antiprotons indicates a partial stopping of nucleons during the collision phase, resulting in an excess of quarks over antiquarks in the fireball region [44. Since baryon number is conserved, this implies the existence of a $T$-dependent baryochemical potential, which in turn translates into a fugacity factor $\lambda_{B}=\exp \left[\frac{\mu_{B}}{T}\right]$ multiplying the entropy density created by baryons. Furthermore, the thermal yield of pions in the fireball is not enough to account for the observed number of pions. This can be compensated by the introduction of a chemical potential for pions (and kaons), which in turn influences the EoS.

By imposing entropy and baryon number conservation, the evolution of $\mu_{B}$ can be followed through 
the fireball expansion. In practice, the evaluation at each $T$ rests on the assumption of the system being an ideal hadronic gas, which we believe is unreasonable near the phase transition. Fortunately, $T_{f} \simeq 100 \mathrm{MeV}$ is far enough distant from the phase transition, and that is where we fix the entropy. Furthermore, we expect pions to be the dominant thermally active degrees of freedom. We use a pion chemical potential $\mu_{\pi}\left(T_{f}\right)=123 \mathrm{MeV}$ as obtained in [45], which gives the correct total pion multiplicity when evaluated with the fireball freeze-out volume.

Correcting the contributions from the different particle species for the corresponding fugacity factors, we obtain a point of the $s(T)$ curve where our interpolation starts. This situation is shown in figure 6 . One observes that, unlike the RHIC scenario, the entropy density under SPS conditions is larger in the range $T_{f}<T<T_{C}$, resulting in a faster dropping of temperature during the hadronic expansion phase.

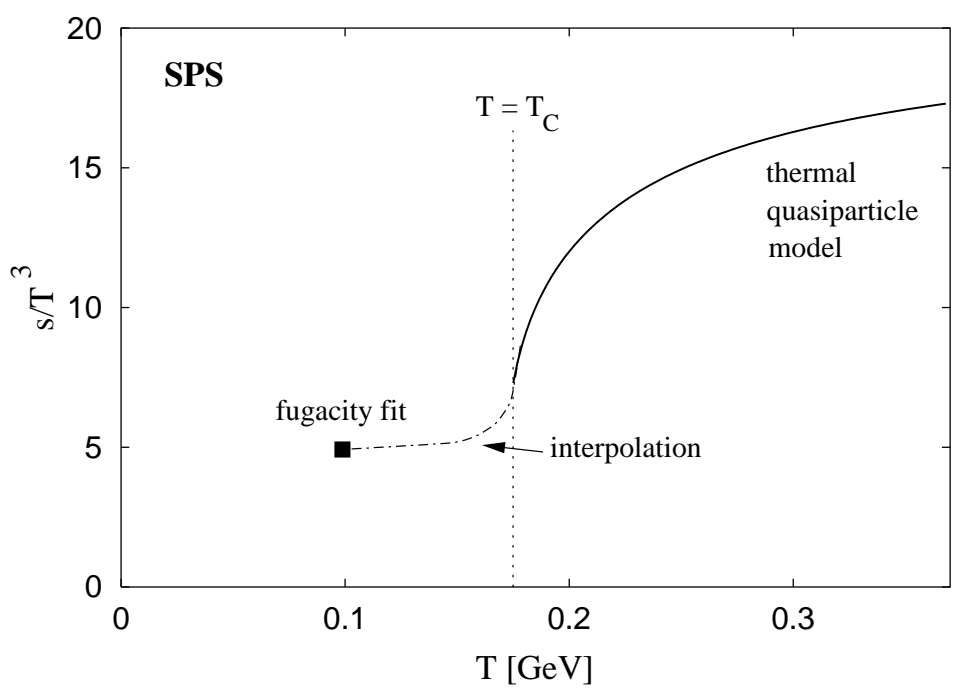

Figure 6: . Temperature dependence of the entropy density for SPS conditions, including the interpolation to the fugacity corrected value at freeze-out. As the entropy density below $T_{f}$ is irrelevant for the fireball evolution, the interpolation is stopped at this point.

Having now specified the relevant part of the EoS of the system, the temperature evolution of the fireball is uniquely determined by the total entropy $S_{0}$ and the evolution of the volume $V(\tau)$ with proper time. The total entropy can be obtained by measuring charged particle multiplicities $N^{+}$and $N^{-}$in suitable rapidity bins and calculating

$$
D_{Q}=\frac{N^{+}-N^{-}}{N^{+}+N^{-}} .
$$

The quantity $D_{Q}$ stands for the inverse of the specific entropy per net baryon $S / B$, and the product $D_{Q}(S / B)$ roughly measures the entropy per pion [50]. For SPS collisions at $160 \mathrm{AGeV}$, we find $S / B=26$ for central collisons. For RHIC $6 \%$ central Au-Au collisions at $130 \mathrm{AGeV}$, the specific entropy $S / B=130$ is substantially higher due to the larger particle multiplicity and the smaller net baryon content in the central region. For beam energies of $200 \mathrm{AGeV}$ that are of interest here, not enough information on $d N / d y$ spectra is available at this moment, so we utilize the predictions from a thermal model calculation 466. With the ratios $\bar{p} / p=0.75, \bar{p} / \pi^{-}=0.09$ and $K^{-} / \pi^{-}=0.15$, we obtain the specific entropy as $S / B \approx 250$, as already estimated (albeit with different parameters) in 43 . 


\subsection{Variations with beam energies and centralities}

It is mainly the detailed information on the final and initial state which enables us to reconstruct the fireball evolution in the case of the $160 \mathrm{AGeV}$ central collision scenario. Unfortunately, no such detailed analysis is available for the $30 \%$ central $\mathrm{PbAu}$ collision scenario for which dilepton data have been taken. The same holds for the $40 \mathrm{AGeV}$ dilepton data. It is therefore necessary to extend the framework established so far to different centralities and beam energies by a suitable re-scaling of key quantities characteristic of the evolution.

First of all, the total entropy deposition in the fireball region must be different when going to more peripheral collisions or lower beam energies. We assume that the entropy per baryon scales with the number of negatively charged hadrons observed in the final state and take the SPS value of $s / \rho_{B}=26$ as a baseline. When going to peripheral collisions, we assume that this value is still a good estimate. Here, the total entropy in the system is naturally reduced because the number of nucleons participating in the collision is smaller.

Second, the overlap geometry is different in peripheral collisions, resulting in a smaller initial fireball volume. Here, we neglect details of the transverse geometry and keep parametrizing the fireball volume as a cylinder. Its initial transverse area is adjusted to the value of the calculated overlap area, hence the cylinder radius is reduced as compared to central collisions.

For the $40 \mathrm{AGeV}$ data, we take the total entropy to be about half the entropy at $160 \mathrm{AGeV}$ because $N_{\pi^{-}}^{160} \approx 600$ and $N_{\pi^{-}}^{40} \approx 310$. The initial energy density, estimated by Bjorken's formula from $d N / d y$ at midrapidity, is about $2 / 3$ of the value at $160 \mathrm{AGeV}$, but still well above the critical energy density required to form a QGP plasma. Looking at the final state of the fireball, HBT analyses of NA49 [52] indicate that the radius parameters are very similar at 40 and $160 \mathrm{AGeV}$, suggesting that the reaction dynamics do not significantly change in this energy region.

Changes in the initial entropy deposition result in a correspondingly different fireball evolution and in general a different freeze-out state. Freeze-out occurs when the mean free path of particles exceeds the dimensions of the fireball. As pions are the most abundant particle species in the fireball, we assume that the pion density determines the mean free path $\lambda$ of particles in the medium. The freeze-out condition reads therefore

$$
\sigma \lambda \rho_{\pi}=1,
$$

where $\sigma$ stands for a typical hadronic cross section. As the observed HBT radii for 40 and $160 \mathrm{AGeV}$ appear rather similar, we do not expect the total freeze-out volume to change more than a factor two. This is thermodynamically consistent with fixing the freeze-out temperature $T_{f}=100 \mathrm{MeV}$ for all SPS scenarios. As $\lambda$ scales $\sim \sqrt[3]{V}$, this is a sensible ansatz - the resulting freeze-out pion densities are very similar when looking at (27). This is still true if we take modificatios of the pion density by a pion chemical potential $\mu_{\pi}$ into account - about the same value of $\mu_{\pi}$ is needed in all SPS scenarios to account for the observed total pion yield (see section 5.7).

The situation is different at RHIC. Here, no large pion chemical potential appears to be necessary to account for the observed total yield and therefore the thermal pion density at a given temperature is lower, leading to a higher freeze-out temperature with (27). We find that an expansion scenario with $T_{f}$ $=130 \mathrm{MeV}$ fits both the observed total particle yield and leads to the correct freeze-out pion density. The freeze-out volume has now to be adjusted accordingly. In order to do this consistently, we simultaneously modify both the final state flow velocities and the fireball radius as compared to our standard scenario. As these quantities result to a first approximation from an accelerated motion, reducing the radial flow by a factor $f$ implies a reduction of the longitudinal flow difference between initial and final state by the same factor $f$ and a reduction of the freeze-out radius by $f^{2}$. Note that a combination of decreasing geometrical radius and flow velocity tends to keep the HBT radius constant. 
The fireball evolution is now calculated as described above, using re-scaled total entropy, initial radius, final state longitudinal and transverse flow and freeze-out radius as new inputs. The parameter sets obtained for the different fireball scenarios are summarized in the appendix.

\subsection{Results and discussion}

The resulting volume evolutions for 40 and $160 \mathrm{AGeV}$ are plotted in figure 7. Note that these curves correspond to the volume at proper time $\tau$, which is larger than the geometrical size of the fireball in the c.m. or the lab frame. The temperature evolution is shown in figure 8. We observe that the QGP

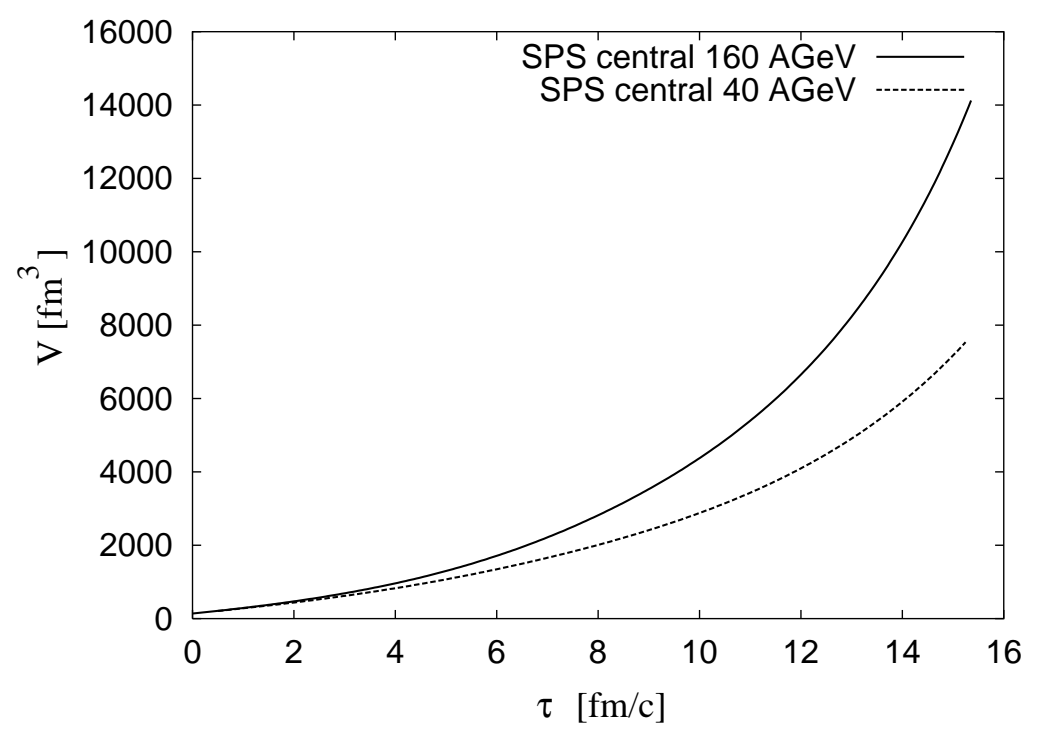

Figure 7: The volume expansion for SPS conditions. Shown are the curves for central $160 \mathrm{AGeV}$ collisions (solid line) and central $40 \mathrm{AGeV}$ collisions (dashed line).

phase lasts about half of the total fireball lifetime for $160 \mathrm{AGeV}$, and considerably less for $40 \mathrm{AGeV}$. As the fireball evolution resulting from our model differs somewhat from the results obtained by other groups, we feel that some clarifying remarks are in order.

In our quasiparticle model there is no mixed phase of coexisting hadronic gas and QGP. Based on the observation that there is no strong first order transition visible in the lattice data, the EoS right and left of $T_{C}$ match smoothly. Inserting the EoS of an ideal quark-gluon gas instead, a large gap in the $s(T)$ diagram is created. The resulting latent heat $(\Delta S) T_{C}$ generates a mixed phase of considerable duration $(5-10 \mathrm{fm})$, as found in previous approaches.

By construction of the model, hadronic observables derived from the expanding fireball, e.g. rapidity distributions of charged particles, $\mathbf{p}_{t}$-spectra, HBT radii and observed particle numbers, are described adequately. This is an important constraint of the model. There is very little freedom left to tune the fireball evolution. Arguably, the EoS inferred from the quasiparticle model is the weakest point in the chain of arguments leading to the complete picture. However, bulk quantities such as freeze-out proper time and freeze-out volume are hardly influenced by the EoS or the phase transition temperature. They can be found even by assuming constant acceleration at all times.

The initial temperatures are quite large ( $\sim 300 \mathrm{MeV}$ for SPS central conditions, $420 \mathrm{MeV}$ for RHIC) and are uniquely determined by the total entropy, the initial volume and our EoS for the quark-gluon 

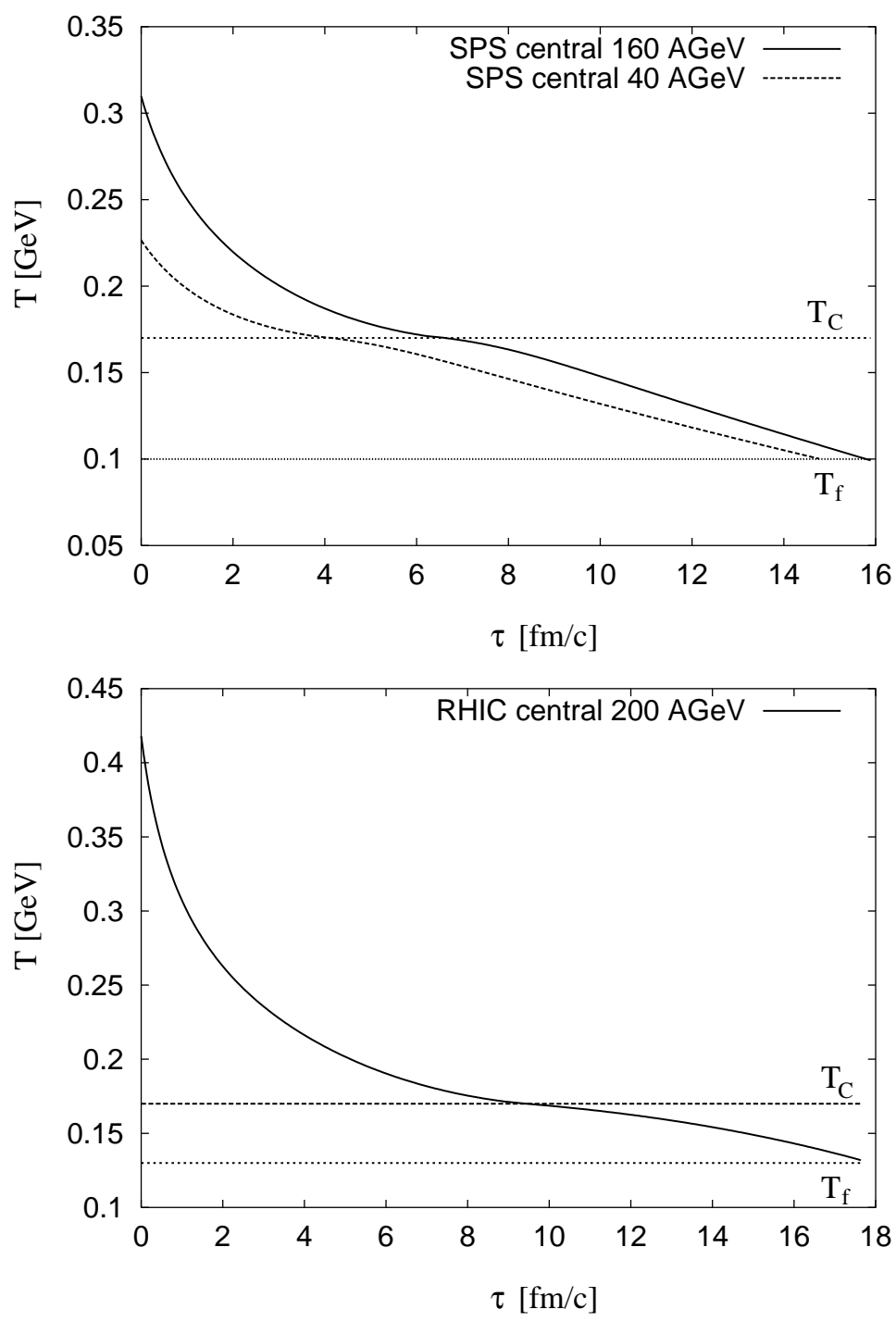

Figure 8: Time evolution of the temperature for SPS and RHIC conditions as obtained with the fireball model described in this work. The critical and freeze-out temperatures are indicated by the horizontal lines.

phase. To some extent these large values related to our choice of the formation time $\tau_{0}=1.0 \mathrm{fm} / \mathrm{c}$ (SPS) and $\tau_{0}=0.6 \mathrm{fm} / c$ (RHIC) of the thermal system; if $\tau_{0}$ is increased, the initial temperature decreases correspondingly. However, unlike the results found in [43], we do not observe any strong sensitivity of the dilepton yields to the choice of $\tau_{0}$ : as the fireball expands, differences in the initial volume become increasingly unimportant. If the total evolution time of the QGP phase is small, these changes may well matter, but as the lifetime of the QGP phase in our model is comparatively large, the possible difference affects only a small fraction of the total lifetime in a region where the fireball volume (and, correspondingly the dilepton yield) is small anyway.

Comparing with the Bjorken estimate of the initial energy density, one should keep in mind that the extrapolation from the final to the initial state is different in the present approach. In the Bjorken 
scenario, no longitudinal acceleration is present, therefore the mapping of final state rapidity distributions to initial state spatial distributions finds a larger initial volume than our scenario. If we assume no or only small longitudinal acceleration in order to compare the two approaches, we find initial temperatures between 220 and $240 \mathrm{MeV}$, consistent with the Bjorken estimate. As this assumption is incompatible with a freeze-out at $100 \mathrm{MeV}$ and the measured freeze-out geometry, we disregard it.

We observe a prolonged lifetime of the QGP evolution phase of the fireball as compared to the results obtained by other groups. Recall that the apparent lifetime of the QGP, as observed in the lab frame, is larger than its lifetime in proper time $\tau$. This is a consequence of our volume evolution and the use of the more realistic EoS of our quasiparticle model, as opposed to that of the ideal quark-gluon gas. Near the phase transition, the entropy density in the quasiparticle model is about a factor two smaller than the one of the ideal quark-gluon gas, and it takes a correspondingly larger volume (and larger evolution time) to reach this entropy density in an isentropic expansion.

The slower cooling time in the hadronic phase of the RHIC scenario as compared to SPS conditions can in essence be traced back to the fact that the entropy density under SPS conditions is enhanced by the fugacity factors whereas this is not such an important effect for RHIC.

\subsection{Chemical composition}

As already mentioned, the total thermal pion yield of the fireball at freeze-out is not enough to account for the observed number of pions, and a large chemical potential of $\mu_{\pi}=123 \mathrm{MeV}$ has to be used to compensate 455. The common point of view is that particle numbers are fixed at a chemical freeze-out point [14] and the absence of inelastic reactions introduces then a chemical potential. The statistical model is enormously succesful in describing the finally observed ratio as a fit of chemical freeze-out temperature and baryon density, using the free properties of particles. However, in the hot and dense environment of a fireball, in medium modifications of particle masses and widths are likely to occur, changing the amount of particle production at the freeze-out temperature. This is not in direct contradiction to the apparent success of the statistical model, as a different set of $T$ and $\mu$ might be able to explain the observed ratios once the modifiactions are taken into account. Furthermore, the absence of all inelastic reactions, leading to decay processes only, is most likely an oversimplification for some particles, e.g. for the $\rho \leftrightarrow \pi \pi$ system. If one knew the particle properties and the particle abundancies at hadronization reliably, the correct way to proceed would be to set up a system of coupled rate equations and follow the various decay and recombination processes to the thermal freeze-out. Unfortunately, theoretically there is no way to assess these properties reliably near the phase transition, as this is a nonperturbative problem. Keeping these issues in mind, we will therefore parametrize the hadrochemistry of the fireball on a phenomenological basis instead of aiming for a detailed solution of the problem.

We adopt the following approach: A pion chemical potential is introduced ad hoc and fitted at the thermal freeze-out point $\left(\mu_{\pi}=123 \mathrm{MeV}\right.$ [45]) to the total pion multiplicity. It is assumed to decrease with temperature linearly up to $T_{C}$ where it vanishes, corresponding to a situation where resonance decays continuously feed pions into the system. All other necessary chemical potentials are adjusted such as to reproduce the observed pion to particle ratios at all times.

\section{Dilepton invariant mass spectra}

Once the time evolution of the fireball is given in terms of the temperature $T(\tau)$, the baryon density $\rho(\tau)$ and the volume $V(\tau)$, and with the knowledge of the photon spectral function, we have all the necessary ingredients to calculate dilepton rates using eq. (3). We fold the result with the acceptance of the CERES and the PHENIX detectors, respectively, and average over the rapidity region covered by 
these two experiments. The so-called 'hadronic cocktail', dileptons produced after freeze-out through various decay processes, with the exception of vector-meson decays, is then added. This contribution fills the region of very low invariant masses $(M<150 \mathrm{MeV})$. The dilepton yields resulting from direct vector meson decays after freeze-out, as described in subsection 4.3, and the Drell-Yan yield from subsection 4.4 are added to the hadronic cocktail, taking into account the limited kinematic acceptance and resolution of the detector.

\subsection{SPS data at 40 and $160 \mathrm{AGeV}$}

We start with a discussion of the SPS CERES/NA45 experiment, treating $40 \mathrm{AGeV}$ and $160 \mathrm{AGeV}$ data in parallel. The final results for the dilepton invariant mass spectra are shown in figs. 9 and 10.

Our calculation reproduces the overall spectrum of the $160 \mathrm{AGeV}$ CERES data quite well. It overestimates the rates somewhat around invariant masses of 200 to $400 \mathrm{MeV}$ and achieves a good description in the region above $400 \mathrm{MeV}$ up to $1.8 \mathrm{GeV}$. Recall that our QGP model rate constitutes only a lower limit on the actual rate because it neglects the radiation from non-partonic (cluster) degrees of freedom above the critical temperature. Bearing in mind that the region above $1 \mathrm{GeV}$ is mainly populated by dileptons originating from the QGP phase, as evident from the left panel of figure 9, there might still be additional radiation close above $T_{C}$ arising from hadronic clusters embedded in a QGP environment. The Drell-Yan contribution is non-negligible, but still outshined by the QGP by a factor of 3 .

Changes in the spectra of the vector mesons indicate tendencies towards chiral symmetry restoration, so the right panel of figure 9 shows the contributions of $\rho, \omega$ and $\phi$ mesons separately, not including the after freeze-out yield. The $\rho$ meson loses its quasiparticle structure entirely due to strong collision broadening at finite density, and fills the whole low mass region between the two pion threshold and $\sim 800 \mathrm{MeV}$. The $\omega$ meson, a sharp peak in the vacuum, broadens at finite temperature due to the thermal scattering process $\omega \pi \rightarrow \pi \pi$. Furthermore, the mass shift at finite baryon density smears the remaining peak structure considerably. Effectively, the remaining signal arises from the direct decays of $\omega$ mesons after freeze-out. The $\phi$ has become a spread-out but still visible resonance structure, showing only moderate broadening at finite temperature and baryon density. It might therefore be a suitable candidate for gauging the strength of vector meson modification.

To test the modelling of the vector meson spectra, we calculate the total number of $\omega$ and $\phi$ meson, suitably averaged over their medium-induced spread in invariant mass, as

$$
\left\langle N_{\omega}\right\rangle=\frac{1}{N_{c h}} \int_{0.65 \mathrm{GeV}}^{0.9} d M \frac{d^{2} N_{\omega \rightarrow e e}}{d \eta d M}=9 \cdot 10^{-7}
$$

and

$$
\left\langle N_{\phi}\right\rangle=\frac{1}{N_{c h}} \int_{0.95 \mathrm{GeV}}^{1.1 \mathrm{GeV}} d M \frac{d^{2} N_{\phi \rightarrow e e}}{d \eta d M}=1.8 \cdot 10^{-7} .
$$

Comparing with numbers from a statistical model calculation, $\left\langle N_{\omega}\right\rangle=4 \cdot 10^{-7}$ and $\left\langle N_{\phi}\right\rangle=2.2 \cdot 10^{-7}$ [04], we indeed find reasonable agreement. Note that the relatively large $\omega$ meson yield is primarily caused by the pion fugacity factor $\left[\exp \left(\mu_{\pi} / T_{f}\right)\right]^{3}$ at freeze-out, which reflects the enhanced feeding through the $3 \pi \rightarrow \omega$ process.

Going from $160 \mathrm{AGeV}$ to $40 \mathrm{AGeV}$ beam energy, analyses of HBT radii and transverse radial flow [52] indicate that the reaction dynamics do not change dramatically, therefore we do not expect drastic differences in the rate. Indeed, the data at $40 \mathrm{AGeV}$ look similar to the $160 \mathrm{AGeV}$ case, and the calculated rate, shown in figure 10, also bears this similarity and achieves a good fit without tuning 

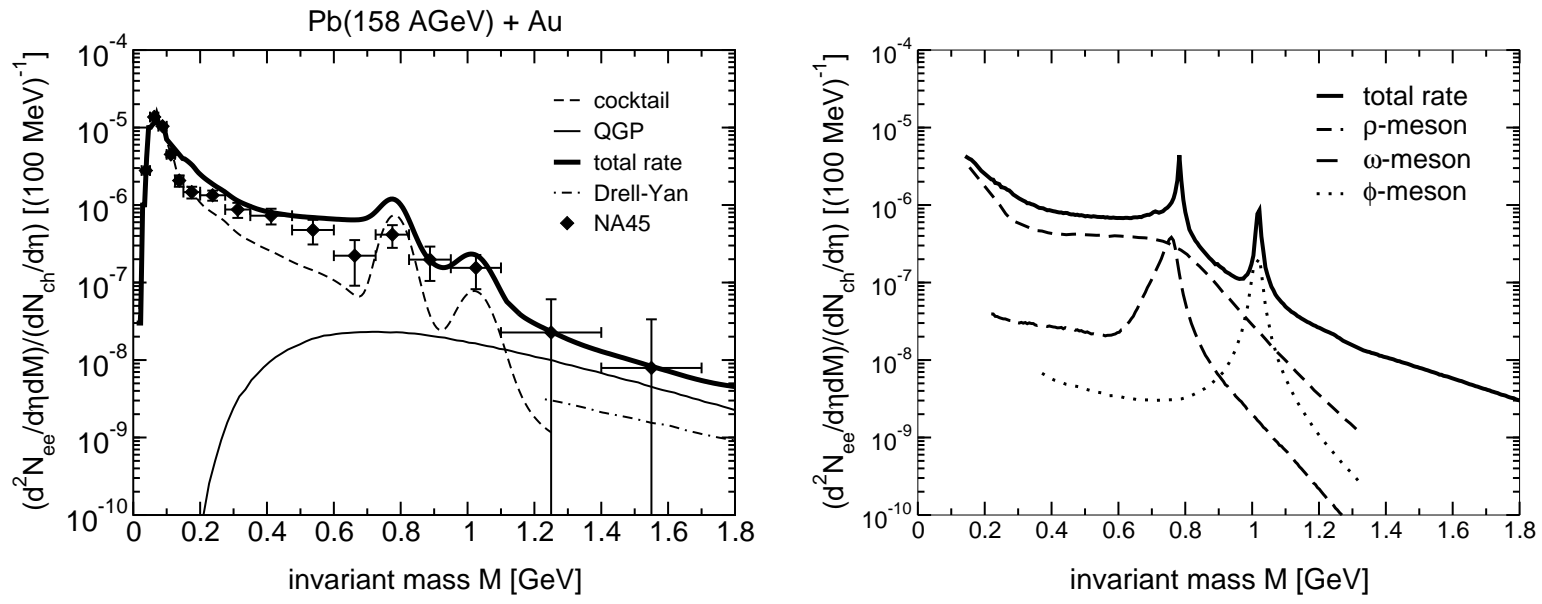

Figure 9: Left: Dilepton invariant mass spectra (see eq. (3)), normalized to $d N_{c h} / d \eta=250$, in units of $(100 \mathrm{MeV})^{-1}$, for the SPS CERES/NA45 Pb(158 AGeV)+Au experiment [55]. Shown are the data, the total rate, the cocktail contribution including the after freeze-out decays of vector mesons, the QGP contribution and the Drell-Yan yield. Right: Contributions from $\rho$-, $\omega$ - and $\phi$-mesons (excluding after freeze-out yield) shown separately, assuming perfect detector resolution.
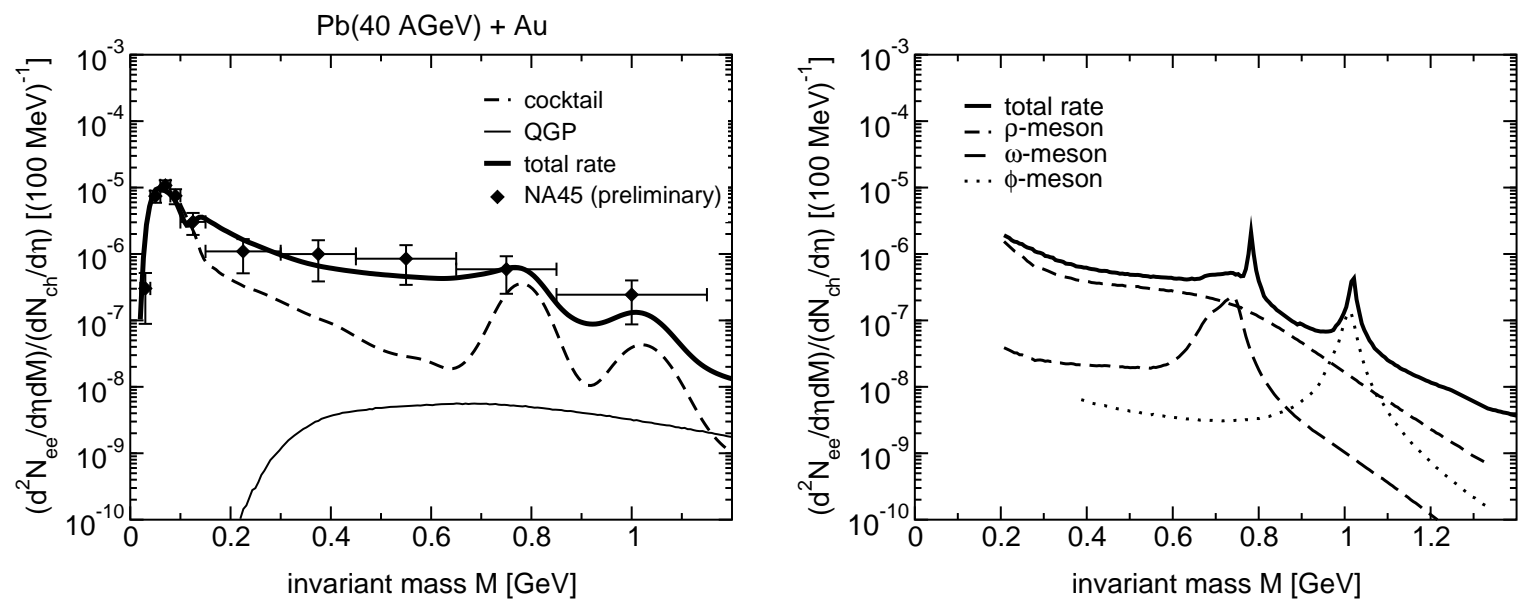

Figure 10: Left: Dilepton invariant mass spectra (see eq. (3)), normalized to $d N_{c h} / d \eta=210$, in units of $(100 \mathrm{MeV})^{-1}$, for the SPS CERES/NA45 $\mathrm{Pb}(40 \mathrm{AGeV})+\mathrm{Au}$ experiment [56]. Shown are the preliminary data, the total rate, the cocktail contribution including the after freeze-out decays of vector mesons and the QGP contribution. Right: Contributions from $\rho$-, $\omega$ - and $\phi$-mesons (excluding after freeze-out yield) shown separately, assuming perfect detector resolution.

the setup of the model. Since the initial temperature is lower and the QGP phase shorter in the 40 AGeV case, the partonic dilepton contribution is obviously much smaller, but nevertheless still present. Owing to the greater initial baryonic density, the in-medium modifications of the vector mesons become more pronounced, most prominently visible in the $\omega$ meson channel. Its downward mass shift drags the 
peak structure along the time evolution of the fireball, creating a small bump on top of the completely dissolved $\rho$ meson that fills up the low-mass region again. Its yield after freeze-out constitutes a visible signal that may be experimentally observable with suitable energy resolution. The $\phi$ meson contribution clearly sticks out above the smooth $\rho$ meson 'continuum'. To conclude, we find no distinct differences in our calculation for the two beam energies probing dilepton production at SPS so far, in accord with experimental findings. This indicates that the general setup of our model is fairly robust. Future data at 20 and $80 \mathrm{AGeV}$ will aid to test this statement.
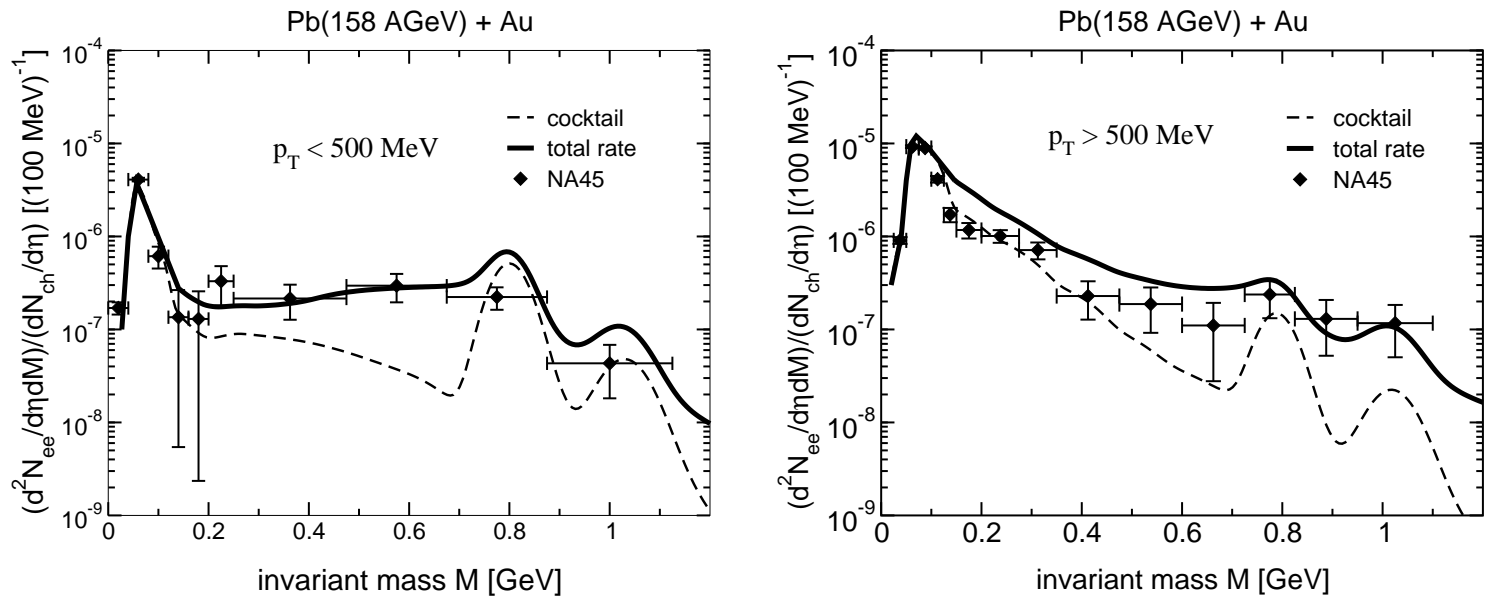

Figure 11: Left: Dilepton invariant mass spectra for transverse momenta of the $e^{+} e^{-}$pair $\mathbf{p}_{t}<500 \mathrm{MeV}$ for the SPS CERES/NA45 $\mathrm{Pb}(158 \mathrm{AGeV})+\mathrm{Au}$ experiment [55]. Shown are the data, the total rate and the cocktail contribution. Right: Same for $\mathbf{p}_{t}>500 \mathrm{MeV}$.
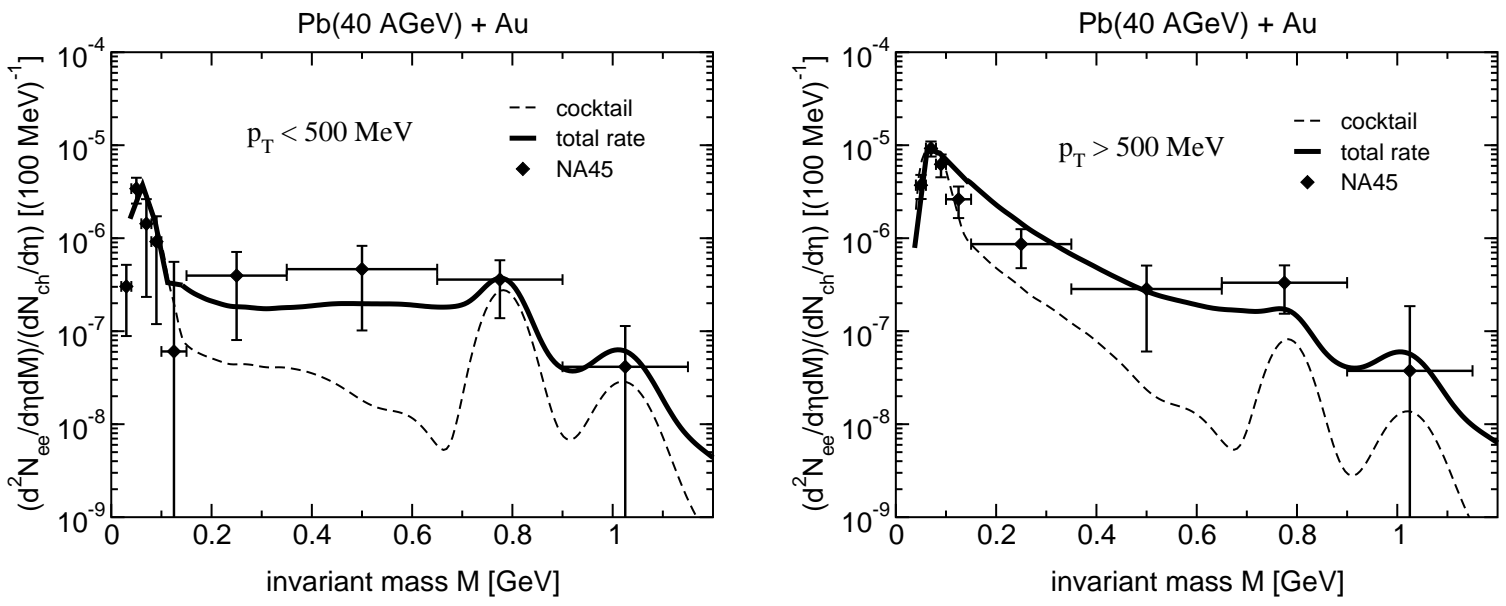

Figure 12: Left: Dilepton invariant mass spectra for transverse momenta of the $e^{+} e^{-}$pair $\mathbf{p}_{t}<500$ $\mathrm{MeV}$ for the SPS CERES/NA45 $\mathrm{Pb}(40 \mathrm{AGeV})+\mathrm{Au}$ experiment [57]. Shown are the data, the total rate and the cocktail contribution. Right: Same for $\mathbf{p}_{t}>500 \mathrm{MeV}$.

The fact that we moderately overestimate the data in the region between 200 and $300 \mathrm{MeV}$ invariant mass 
requires a comment. Since this range is dominated by the low-mass behaviour of the $\rho$ meson spectral function at finite density, this behaviour may indicate that the influence of finite three-momentum on the spectra in that very region is non-negligible. Consider the $160 \mathrm{AGeV}$ and $40 \mathrm{AGeV}$ data taken for different transverse momenta $\mathbf{p}_{t}<500 \mathrm{MeV}$ and $\mathbf{p}_{t}>500 \mathrm{MeV}$, shown in figures 11 and 12 . We observe that the general shape of the data pattern is well described by the calculation for both $\mathbf{p}_{t}$ regions and both beam energies. However, for the high $\mathbf{p}_{t}$ case, the calculation again overshoots the data in the low mass region whereas this is not so in the low $\mathbf{p}_{t}$ case. This can be traced to the use of the spectral functions for three-momentum equal to zero in order to describe the photon spectral function in the hadronic phase. This approximation is of limited validity at high $\mathbf{p}_{t}$ where the spectral function ought to become smaller than in our approximation. Therefore we expect improved agreement with the data for the low invariant mass region both at 40 and $160 \mathrm{AGeV}$ once this effect is taken into account properly.

Our setup of the fireball model enables us to gain additional detailed insight into the time evolution of the dilepton yield. This is demonstrated in figure 13. Here the different stages of the fireball evolution leave distinct marks in the time-resolved dilepton yield. For early times, dileptons come entirely from the $q \bar{q}$ quasiparticle annihilation processes. The movement of the invariant mass threshold reflects the temperature dependence of the quasiparticle mass which decreases near the phase transition at $\tau \sim 7 \mathrm{fm} / \mathrm{c}$. One observes that, in spite of the growing fireball volume, the contributions from later timeslices to the total yield become progressively less important until the hadronic phase takes over at $\tau>7 \mathrm{fm} / \mathrm{c}$. This surprising behaviour is enforced by the confinement factor $C(T)$ which reduces the thermodynamically active degrees of freedom significantly near the phase boundary. The system then enters the hadronic evolution phase without going through a mixed phase. The most prominent feature is the rapid filling of the low invariant mass region through the density-broadened $\rho$ meson spectrum which ends up as an enhanced pion continuum. The $\phi$ meson starts contributing its characteristic peak and, as the system cools down further, the $\omega$ meson begins to emerge, albeit as a broad structure. Note that while the hadronic contributions fill primarily the low invariant mass region, their yield above 1 $\mathrm{GeV}$ is negligible in this late evolution phase.

\subsection{RHIC at $\sqrt{s}=200 \mathrm{AGeV}$}

For the RHIC scenario, thermally generated dileptons are dominant. Measurements of proton ratios at $\sqrt{s}=130 \mathrm{AGeV}$ indicate that the central collision region remains almost net-baryon free, compared to SPS energies. Within a statistical thermal model, the particle ratios are accounted for by a small baryon chemical potential of about $50 \mathrm{MeV}$ at chemical freezeout [46]. At $200 \mathrm{AGeV}$, this value is predicted to be even smaller. Effects of finite baryon density are therefore almost absent and consequently both the $\rho$ and the $\phi$ are expected to show up in the spectrum as pronounced structures, whereas there should be no clear trace of the in-medium $\omega$ due to its strong thermal broadening. Contributions from Drell-Yan processes, which dominate in the very high invariant mass region, are an order of magnitude smaller.

The corresponding prediction for the dilepton yield at $200 \mathrm{AGeV}$, including the schematic acceptance for the PHENIX detector, is shown in figure 14. The $\omega$ and $\phi$ meson resonances clearly stick out over the smooth $\rho$ meson and QGP contributions. Although PHENIX will only start to measure at $M \geq 1 \mathrm{GeV}$, it may be possible to resolve the $\phi$ meson peak. However, a significant part of the peak strength is built up by the after freeze-out contributions, making it difficult to disentangle the in-medium modifications on the hadrons. For $M \geq 1.3 \mathrm{GeV}$, the dilepton spectrum is dominated by thermal QGP radiation, outshining the hard Drell-Yan dilepton yield.

Comparing our prediction for PHENIX with the one shown in ref. [43], we find rough agreement of the rate for the low mass region below $\sim 1 \mathrm{GeV}$. Although the dilepton yield from the QGP phase is suppressed in our case by the factor $C(T)^{2}$ (cf. section 4.1), we still find an enhancement of a factor of about 4 in the range $1.3-2.5 \mathrm{GeV}$ over the rate in 43 that employs a (perturbative) chemical undersaturation model in the QGP phase. Owing to the non-perturbative nature of the QGP close to 


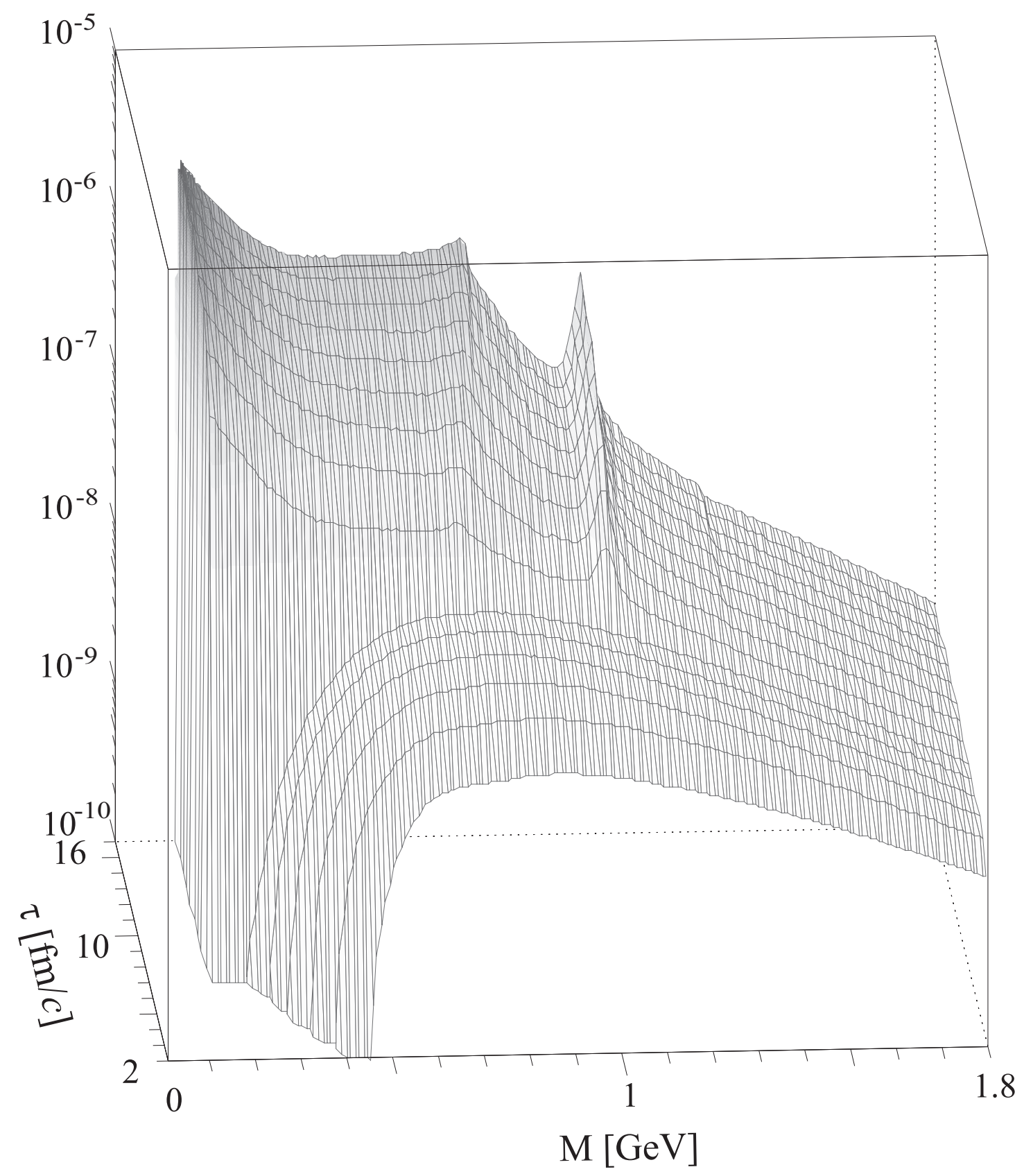

Figure 13: Time evolution of the (integrated) dilepton yield for the SPS scenario. The vertical scale is the same as in fig. 9. The cocktail contribution has not been included in this picture, and perfect resolution was assumed.

$T_{C}$, this model may not be applicable in that very region. Note that our rate also constitutes only a lower limit there, so that the actual rate may even be larger. High precision data will allow to pin down one or the other model. 

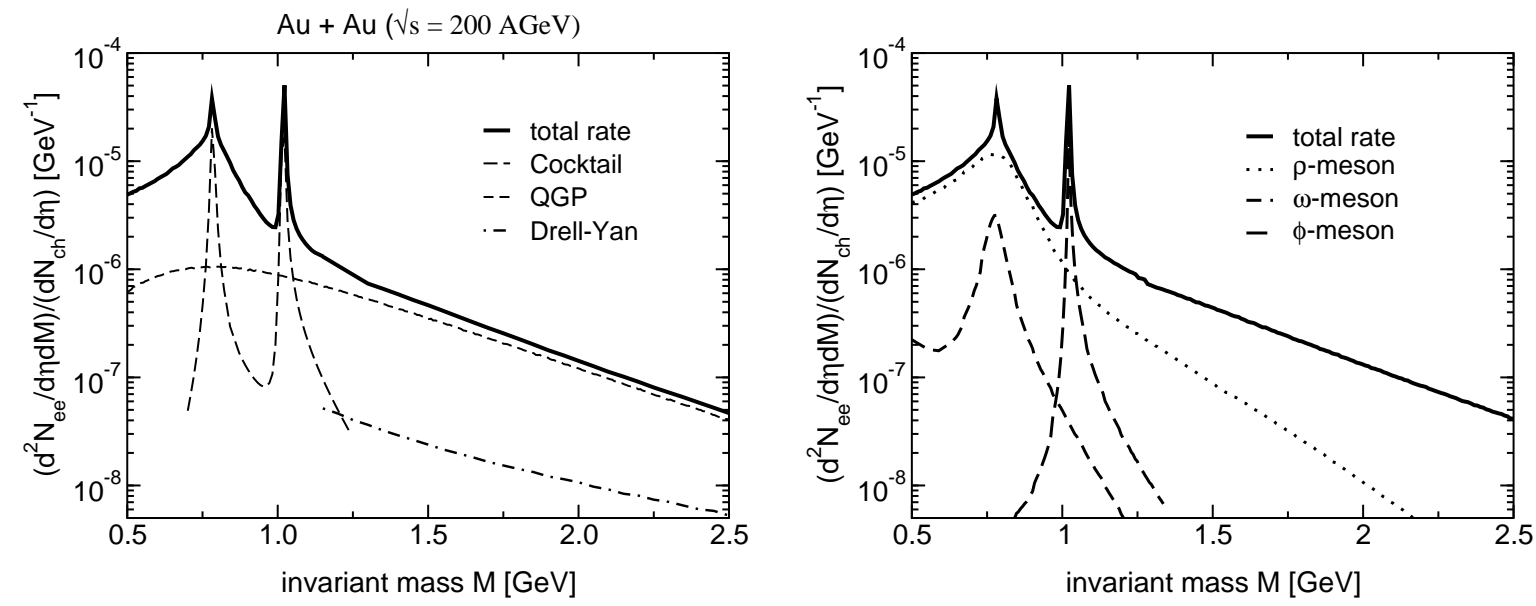

Figure 14: Left: Dilepton invariant mass spectra, normalized to $d N_{c h} / d \eta=650$ [53], in units of $\mathrm{GeV}^{-1}$, for the RHIC experiment PHENIX at $\sqrt{s}=200 \mathrm{AGeV}$. Shown are the total rate, the cocktail consisting of the after freeze-out decays of $\omega$ and $\phi$ vector mesons, the QGP contribution and the Drell-Yan yield. Right: Contributions from $\rho$-, $\omega$ - and $\phi$-mesons (excluding after freeze-out yield) shown separately.

\subsection{Sensitivity to model parameters}

We would like to stress that the gross features of our model are set, once the parametrization of the fireball evolution has been matched to the hadronic observables and the EoS of both phases has been constructed in accordance with lattice QCD and empirical constraints. The remaining uncertainties, mainly about the initial state of the fireball, the thermal masses of the quasiparticles and the detailed shape of the EoS, do not alter the results substantially; they lead to only moderate or even weak dependence on those parameters. Fine-tuning is still possible, but only within the limits that retain consistency with the overall framework.

We have investigated the sensitivity of the model to parameter changes in some detail for the SPS scenario at $160 \mathrm{AGeV}$. In order to get a theoretical 'error band', we investigated the extremes of our parameter ranges, a combination of parameters that yields the largest and the smallest possible QGP contribution. The resulting range is shown in figure 15 as a grey band, together with the data points and the curve from the previous section.

Regarding the initial conditions, the largest uncertainty comes from the initial fireball formation length $z_{0}$ or, equivalently, the time $\tau_{0}$. We let it vary from $0.5 \mathrm{fm} / c$ to $2 \mathrm{fm} / c$, i.e. from fast to slow equilibration. Consider next the initial longitudinal flow velocity $v_{z}^{i}$. A variation of this quantity from $0.3 c$ to 0.8 $c$ implies a modification of the pressure-driven accelerated motion in order to arrive at the same final velocity of $0.9 c$, as determined by the rapidity distributions of the observed hadrons. Strong deviations from $v_{z}^{i} \simeq 0.5 c$, however, lead to inconsistent values for the thermal freeze-out temperature $T_{f}$. Finally, modifications of the critical temperature $T_{C}$ influence mainly the relative weight of the contributions from the QGP phase and the hadronic gas phase to the dilepton yield and hence change the shape of the dilepton spectra. Lattice data on $T_{C}$ suggest a range from $140 \mathrm{MeV}$ for three massless, thermally active flavours to about $185 \mathrm{MeV}$ for two massless flavours and a realistic pion mass. Due to the self-consistent modelling of the temperature and volume evolution, there is no simple one-to-one correspondence of these parameters to the dilepton yield.

The upper limit of the grey band in figure 15 now corresponds to the scenario with a small QGP 


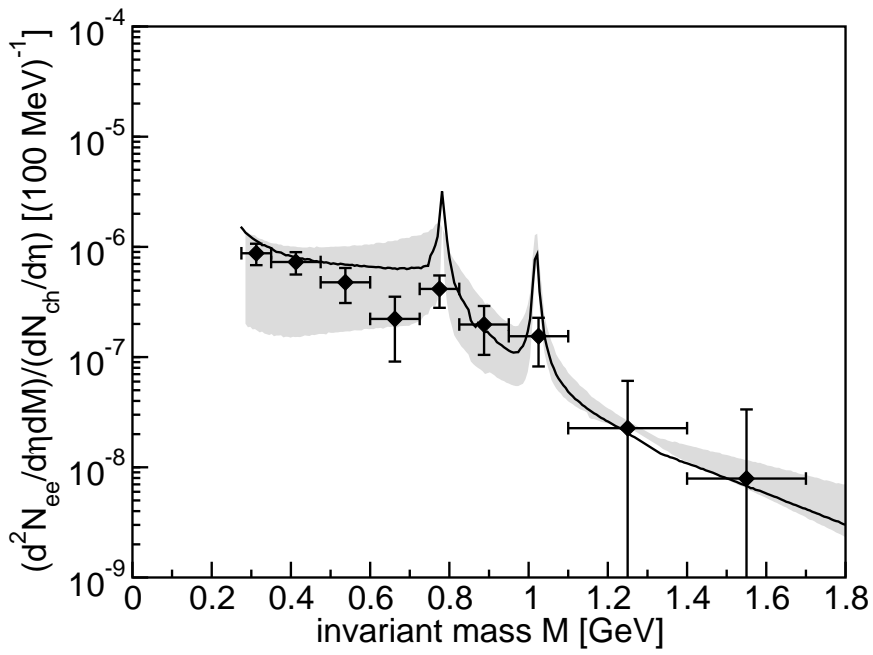

Figure 15: Dilepton invariant mass spectra for the SPS CERES/NA45 experiment at $160 \mathrm{AGeV}$. Shown are data (symbols), the total rate introduced in section 6.1 (solid line) and a band (shaded) that represents the range in the dilepton yield when varying the parameters $\tau_{0}, v_{z}^{i}$ and $T_{c}$. See text for details.

contribution, i.e. small $\tau_{0}$, large $v_{z}^{i}$ and high $T_{C}$. On the other hand, the lower limit includes a large QGP contribution with large $\tau_{0}$, small $v_{z}^{i}$ and low $T_{c}$. It is instructive to note that the shape of the spectrum changes only moderately above $1 \mathrm{GeV}$ invariant mass within these extreme parameter variations. The first scenario, however, tends to overestimate the data in the region of the $\rho$ peak, whereas the second scenario does not leave enough time for the hadronic phase to build up the $e^{+} e^{-}$excess in the low-mass region between 200 and $800 \mathrm{MeV}$, effectively ruling out a large QGP contribution.

\section{Concluding Remarks}

We have calculated dilepton radiation from an expanding fireball created in ultra-relativistic heavy-ion collisions over a wide range of beam energies, from SPS $40 \mathrm{AGeV}$ via SPS $160 \mathrm{AGeV}$ to RHIC $\sqrt{s}=$ $200 \mathrm{AGeV}$.

We have explored the evolution of a fireball through the quark-gluon and hadronic phases as they are assumed to be characteristic of the QCD equation of state. Furthermore, we have emphasized the relevance of using input and constraints from lattice QCD thermodynamics in the analysis of dilepton signals from the expanding fireball. The fact that the measured dilepton rates could be reproduced using a fireball model fixed by hadronic information only is a nontrivial confirmation of its validity. Further data for different energies and/or nuclear systems will help to strengthen or weaken this position.

High resolution measurements of the invariant mass spectrum around the region of the $\omega$ and the $\phi$ mass can reveal information on the average density and temperature in the hadronic phase. The broadening and mass shift of the $\omega$ meson with temperature can serve as a 'thermometer' once the cocktail contribution is reliably assessed. A visible enhancement in the $\omega$ region beyond the rate originating from $\omega$-decays after freeze-out would hint at a temperature which is on average lower than assumed in this model and therefore point to a lower freeze-out temperature or alternative in-medium effects on hadrons. 
On the other hand, the $\phi$ meson signal, being nearly unmodified by temperature effects over a comparably long timespan, may be used as a 'standard candle' at RHIC conditions.

Detailed knowledge of the slope of the invariant mass spectrum above $1 \mathrm{GeV}$ gives an indication of the average temperature in the quark-gluon phase, serving as a constraint for the fireball dynamics in the QGP region. Its total normalization is useful to estimate the effects of a possible underpopulation of the quark and gluon phase space in early stages of the evolution, or of phenomenological models of the QGP, such as the confinement model presented in section 3. It is remarkable that this confinement model is able to reproduce the data despite the suppression of dilepton radiation near the phase transition temperature.

Measurements using different nuclear systems or different impact parameter can provide different relative weights of QGP and hadronic dilepton radiation to the observed yield. One may thus hope to disentangle these contributions and test the model assumptions above and below the phase transition separately, at least qualitatively.

The present scenario supports the hypothesis that the quark-gluon phase is actually reached, at a transient stage, in heavy-ion collisions at CERN/SPS and (more pronouncedly) at RHIC. Within our outlined approach, a purely hadronic framework would not be successful in comparison with existing data. The reason is that the relative strength of hadronic contributions to the high mass region and the low mass region is quite different as compared to those of the QGP (cf. figure 9). Purely hadronic scenarios which are able to account for the low mass region would necessarily fail in the high mass region and vice versa. Furthermore, fireball thermodynamics as described in this work leads to temperatures well above $T_{C}$ for a broad range of initial conditions, though not necessarily to significant contributions to the dilepton yield from the high-temperature region. The upcoming measurements at RHIC can be expected to provide further interesting insights.

\section{Acknowledgements}

We are grateful to Ralf Rapp, Jochen Wambach, Francois Arleo, Alberto Polleri, Hans J. Specht and Sanja Damjanovic for stimulating discussions and exchanges in the course of preparing this paper.

\section{A Fireball properties}

In the following, we summarize key properties of the different fireball scenarios discussed in the paper. We characterize each scenario by the proper time for fireball formation, $\tau_{0}$, the phase transition time $\tau_{c}$ and the freeze-out time $\tau_{f}$. Note that the corresponding times in the c.m. frame can be considerably larger, especially for RHIC conditions where volume elements are travelling near the speed of light at the fireball edge. Additionally, we quote the volume at freeze-out and the flow velocities at rms radius $v_{\perp}^{f}$ and maximum longitudinal extention $v_{z}^{f}$. In order to compare the thermodynamic conditions, we provide $s / \rho$, the entropy per baryon.

Note that the expansion is modelled in proper time. The lifetime of the fireball in the RHIC c.m. frame, for example, is more than four times larger due to time dilatation at the fireball edge. 


\begin{tabular}{|l|c|c|c|c|c|c|c|c|}
\hline & $\tau_{0}[\mathrm{fm} / c]$ & $\tau_{c}[\mathrm{fm} / c]$ & $\tau_{f}[\mathrm{fm} / c]$ & $V_{f}\left[\mathrm{fm}^{3}\right]$ & $v_{\perp}^{f}$ & $v_{z}^{i}$ & $v_{z}^{f}$ & $s / \rho_{B}$ \\
\hline \hline SPS 40 AGeV & 1.5 & 4.0 & 15.0 & 7040 & 0.36 & 0.45 & 0.75 & 13 \\
\hline SPS 160 AGeV & 1.0 & 6.5 & 16.0 & 14.400 & 0.53 & 0.45 & 0.9 & 26 \\
\hline RHIC 200 AGeV & 0.6 & 10.0 & 18.0 & 99846.1 & 0.56 & 0.9 & 0.9985 & 250 \\
\hline
\end{tabular}

Table 1: Key parameters of the fireball evolution scenarios

\section{References}

[1] C. Bernard et al., Phys. Rev. D55 (1997) 6881; F. Karsch, E. Laermann and A. Peikert, Phys. Lett. B478 (2000) 447.

[2] F. Karsch, E. Laermann, A. Peikert, Ch. Schmidt and S. Stickan, Nucl. Phys. Proc. Suppl. 94 (2001) 411.

[3] F. Karsch, Nucl. Phys. A698 (2002) 199.

[4] G. Q. Li, C. M. Ko and G. E. Brown, Phys. Rev. Lett. 75 (1995) 4007; G. Q. Li, C. M. Ko and G. E. Brown, Nucl. Phys. A 606 (1996) 568; G. Q. Li, C. M. Ko, G. E. Brown and H. Sorge, Nucl. Phys. A 611 (1996) 539.

[5] W. Cassing, W. Ehehalt and C. M. Ko, Phys. Lett. B363 (1995) 35; W. Cassing and E. L. Bratkovskaja, Phys. Reports 308 (1999) 65.

[6] J. Sollfrank, P. Huovinen, M. Kataja, P. V. Ruuskanen, M. Prakash and R. Venugopalan, Phys. Rev. C 55 (1997) 392.

[7] C. M. Hung and E. Shuryak, Phys. Rev. C57 (1998) 1891.

[8] F. Klingl, N. Kaiser and W. Weise, Nucl. Phys. A606 (1996) 329.

[9] R. Rapp and J. Wambach, Eur. Phys. J. A6 (1999) 415.

[10] R. A. Schneider and W. Weise, Eur. Phys. J. A9 (2000) 357.

[11] C. Zhai and B. Kastening, Phys. Rev. D52 (1995) 7232.

[12] G. Boyd, J. Engels, F. Karsch, E. Laermann, C. Legeland, M. Lutgemeier and B. Petersson, Nucl. Phys. B469 (1996) 419; K. Kajantie, M. Laine, J. Peisa, A. Rajantie, K. Rummukainen and M. Shaposhnikov, Phys. Rev. Lett. 79 (1997) 3130.

[13] K. Geiger, Phys. Reports 258 (1995) 237.

[14] P. Braun-Munzinger, I. Heppe and J. Stachel, Phys. Lett. B465 (1999) 15.

[15] D. K. Srivastava, nucl-th/9903066.

[16] P. Petreczky, F. Karsch, E. Laermann, S. Stickan and I. Wetzorke, Nucl. Phys. Proc. Suppl. 106 (2002) 513.

[17] P. Aurenche, F. Gelis, R. Kobes and H. Zaraket, Phys. Rev. D58 (1998) 085003.

[18] P. Aurenche, F. Gelis and H. Zaraket, Phys. Rev. D61 (2000) 116001.

[19] R.A. Schneider and W. Weise, Phys. Rev. C64 (2001) 055201.

[20] A. Peshier, B. Kämpfer and G. Soff, Phys. Rev. C61 (2000) 045203.

[21] P. Levai and U. Heinz, Phys. Rev. C57 (1998) 1879. 
[22] M. H. Thoma, hep-ph/0010164.

[23] E. Braaten, R. D. Pisarski and T.C. Yuan, Phys. Rev. Lett. 64 (1990) 224.

[24] A. Peshier and M. H. Thoma, Phys. Rev. Lett. 84 (2000) 841.

[25] F. Klingl, N. Kaiser and W. Weise, Z. Phys. A356 (1996) 193.

[26] R. A. Schneider and W. Weise, Phys. Lett. B515 (2001) 89.

[27] C. Song, C. M. Ko and C. Gale, Phys. Rev. D50 (1994) R1827.

[28] G. Q. Li and C. Gale, Nucl. Phys. A638 (1998) 491.

[29] V. L. Eletsky and B. L. Ioffe, Phys. Rev. D47 (1993) 3083; V. L. Eletsky and B. L. Ioffe, Phys. Rev. D51 (1995) 2371.

[30] M. Urban, M. Buballa and J. Wambach, Phys. Rev. Lett. 88 (2002) 042002; E. Marco, R. Hofmann and W. Weise, hep-ph/0110110.

[31] W. A. Zajc et al., PHENIX Collaboration, Nucl. Phys. A698 (2002) 39.

[32] PHOBOS Collaboration, Phys. Rev. Lett. 87 (2001) 102301.

[33] F. Klingl, N. Kaiser and W. Weise, Nucl. Phys. A624 (1997) 527.

[34] A. D. Martin, R. G. Roberts, W. J. Stirling and R. S. Thorne, hep-ph/0201127.

[35] H. L. Lai et al. (CTEQ), Eur. Phys. J. C12 (2000) 375.

[36] M. Glück, E. Reya, and A. Vogt, Eur. Phys. J. C5 (1998) 461.

[37] P. L. McGaughey et al. (E772), Phys. Rev. D50 (1994) 3038.

[38] K. J. Eskola, V. J. Kolhinen and C. A. Salgado, Eur. Phys. J. C9 (1999) 61.

[39] F. Arleo, hep-ph/0201066, to appear in Phys. Lett. B.

[40] S. Gavin, P. L. McGaughey, P. V. Ruuskanen and R. Vogt, Phys. Rev. C54 (1996) 2606.

[41] E. Shuryak, Phys. Rev. C55 (1997) 961.

[42] Z. Lin, R. Vogt and X.-N. Wang, Phys. Rev. C57 (1998) 899.

[43] R. Rapp, Phys. Rev. C63 (2001) 054907.

[44] B. Jacak, NA44 Collaboration, in "Hot and Dense Nuclear Matter", W. Greiner, H. Stöcker and A. Gallmann, eds. (Plenum, New York, 1994), p. 607.

[45] B. Tomasik, U. A. Wiedemann and U. W. Heinz, nucl-th/9907096.

[46] P. Braun-Munzinger, D. Magestro, K. Redlich and J. Stachel, Phys. Lett. B518 (2001) 41.

[47] J. D. Bjorken, Phys. Rev. D27 (1983) 140.

[48] P. Braun-Munzinger and J. Stachel, Nucl. Phys. A638 (1998) 3

[49] G. Agakichiev et al., CERES collaboration, Phys. Rev. Lett. 75 (1995) 1272; G. Agakichiev et al., CERES collaboration, Phys. Lett. B422 (1998) 405.

[50] J. Letessier, A. Tounsi, U. Heinz, J. Sollfrank and J. Rafelski, Phys. Rev. D51 (1995) 3408.

[51] F. Karsch, E. Laermann, P. Petreczky, S. Stickan and I. Wetzorke, hep-lat/0110208.

[52] S. Y. Panitkin, Nucl. Phys. A698 (2002) 323. 
[53] PHOBOS Collaboration, Phys. Rev. Lett. 88 (2002) 022302.

[54] H. J. Specht, private communication.

[55] CERES Collaboration, Nucl. Phys. A661 (1999) 23.

[56] S. Damjanovic for the CERES collaboration, Proceedings of the Fourth International Conference on Physics and Astrophysics of Quark Gluon Plasma (ICPAQGP),Jaipur, 2001, to appear in Pramana, India; see also nucl-ex/0111009.

[57] S. Damjanovic, private communication. 